CONFORMAL GEOMETRY AND DYNAMICS

An Electronic Journal of the American Mathematical Society

Volume 2, Pages 1-24 (February 3, 1998)

S $1088-4173(98) 00009-5$

\title{
THE TEICHMÜLLER DISTANCE ON THE SPACE OF FLAT CONFORMAL STRUCTURES
}

\author{
HIROYASU IZEKI
}

\begin{abstract}
We define the Teichmüller pseudodistance on spaces of flat conformal structures by the same manner as classical Teichmüller distance on the Teichmüller space of Riemann surfaces. We will prove that for compact manifolds this pseudodistance becomes a complete distance. We will also prove similar results for noncompact manifolds under certain assumptions.
\end{abstract}

\section{INTRODUCTION}

The aim of this paper is to show that, on the space of flat conformal structures on a compact manifold $M$, there is a complete distance defined by means of quasiconformal mappings. The distance is the same as the Teichmüller distance on the Teichmüller space when $M$ is an oriented surface. First, we recall some definitions.

Let $M$ be a smooth $n$-manifold and $C$ a conformal class on $M$. If, for any point $p$ of $M$, there exists a metric $g$ in $C$ such that $g$ is flat on some neighborhood of $p$, then $(M, C)$ is called conformally flat. A conformal class on $M$ is called a flat conformal structure if $(M, C)$ is conformally flat. A Riemannian manifold $(M, g)$ is conformally flat if the conformal class containing $g$ is a flat conformal structure; $M$ is said to be conformally flat if $M$ admits a flat conformal structure. A flat conformal structure on an oriented surface $S$ is nothing but a conformal structure of a Riemann surface. A flat conformal structure on a conformally flat manifold can be considered as a natural generalization of a conformal structure on a surface to higher dimensional manifolds. The space of conformal structures on an oriented surface is understood as, for instance, the Teichmüller space of Riemann surfaces. In this paper, we are interested in the space of flat conformal structures on a manifold with $n \geq 3$.

Let $\operatorname{Diff}_{H}(M)$ be a group consisting of the diffeomorphisms of $M$ homotopic to the identity. We consider the space of flat conformal structures defined as follows:

$$
\mathcal{T}(M)=\{\text { flat conformal structures on } M\} / \operatorname{Diff}_{H}(M) \text {. }
$$

We denote by $[C]$ the element of $\mathcal{T}(M)$ represented by a flat conformal structure $C$. The space $\mathcal{T}(M)$ is topologized by the quotient topology which comes from the compact- $C^{\infty}$ topology of the space of Riemannian metrics on $M$. If $n=2$, then this space $\mathcal{T}(M)$ is just the Teichmüller space of $M$, and hence $\mathcal{T}(M)$ can be viewed as a higher dimensional analogue of the Teichmüller space. On the Teichmüller space, there is a complete distance called the Teichmüller distance that is defined

Received by the editors February 24, 1997 and, in revised form, October 24, 1997.

1991 Mathematics Subject Classification. Primary 58D27.

Key words and phrases. Conformally flat manifolds, quasiconformal mappings.

(c)1998 American Mathematical Society 
by using quasiconformal mappings as follows: for two points $\left[C_{1}\right]$ and $\left[C_{2}\right]$ in $\mathcal{T}(M)$ of a surface $M$, the Teichmüller distance between these two points is defined by

$$
d\left(\left[C_{1}\right],\left[C_{2}\right]\right)=\log \inf K\left(\varphi ;\left(M, C_{1}\right),\left(M, C_{2}\right)\right),
$$

where the infimum is taken over all quasiconformal mappings between $\left(M, C_{1}\right)$ and $\left(M, C_{2}\right)$ that are homotopic to the identity, and $K\left(\varphi ;\left(M, C_{1}\right),\left(M, C_{2}\right)\right)$ denotes the maximal dilatation of $\varphi$. This definition of the Teichmüller distance still makes sense for higher dimensional manifolds, and it is easy to see that this gives a pseudodistance on $\mathcal{T}(M)$ (see section 3). We call this pseudodistance the Teichmüller pseudodistance in this paper. If this pseudodistance turns out to be a complete distance, it will be a useful tool for the investigation of the space $\mathcal{T}(M)$. The main result of this paper is the following:

Main Theorem. If $M$ is compact, then the Teichmüller pseudodistance don $\mathcal{T}(M)$ is a complete distance. Moreover, the topology given by d agrees with the original topology of $\mathcal{T}(M)$.

We call the distance $d$ given by this theorem the Teichmüller distance on $\mathcal{T}(M)$. For a noncompact manifold $M$, we can prove that $d$ becomes a distance on a subset of $\mathcal{T}(M)$ if $M$ satisfies a certain condition (see section 6). We cannot say, however, that $d$ is a distance in general. It should be mentioned that we can prove for compact manifolds that $d$ becomes a distance by using works of Lelong-Ferrand ([9], [10]). However, we need another step to prove the completeness and also to prove it for noncompact manifolds.

The proof roughly goes as follows. In order to prove the main theorem, it is essential to show the compactness of bounded sequences with respect to $d$. To do this, we have to consider the compactness in a wider space endowed with a weaker topology, denoted by $\mathcal{T}^{0}(M)$, consisting of flat conformal structures with low regularity; the compactness in this space gives us a candidate for the limit of a subsequence of the given bounded sequence. For this purpose, in section 2, we introduce the notion of $C^{r}$ Möbius structures following [2]; the space $\mathcal{T}^{0}(M)$ is defined to be the Teichmüller space of $C^{0}$ Möbius structures (see Definition 2.2) and $\mathcal{T}(M)$ can be viewed as a subset of $\mathcal{T}^{0}(M)$. By using some basic theorems on quasiconformal mappings listed in section 3, we can prove that the bounded sequences with respect to $d$ are sequentially compact in $\mathcal{T}^{0}(M)$ with its natural topology (Lemma 4.1). Then, in section 5 , we can prove that $d$ is a complete distance on $\mathcal{T}^{0}(M)$ if the topology of $\mathcal{T}^{0}(M)$ is not so pathological (Theorem 5.6). If $M$ is compact, then $\mathcal{T}^{0}(M)$ is sufficiently nice for our purpose, and we conclude that $d$ extended to $\mathcal{T}^{0}(M)$ is a complete distance. Together with a regularity lemma (Lemma 2.4) which is an easy consequence of the holonomy theorem (Theorem 2.3), we see that $d$ is actually a complete distance on $\mathcal{T}(M)$. For the case that $M$ is noncompact, we can prove, under some assumptions, that $d$ is a complete distance on a subset of $\mathcal{T}^{0}(M)$. This will be shown in section 6 .

It should be mentioned that our proof does not work for the case $n=2$. In spite of this fact, in a sense, our proof given in this paper can still be considered as a sort of extension of the proof for the case of Riemann surfaces. We will explain this in section 5. The difference between the case of Riemann surfaces and the higher dimensional case comes from that between the Riemann mapping theorem and Liouville's theorem for conformal mappings. This brings us the lack of nice uniformization of flat conformal structures on higher dimensional manifolds. Of 
course, the developing map associated to a flat conformal structure, equivalently a Möbius structure when $n \geq 3$, can be viewed as a kind of uniformization of a flat conformal structure. However, since developing maps may be very wild in general, our situation is more complicated than the case of Riemann surfaces.

In section 7 , we consider the existence of extremal mappings. It was an important problem in the classical theory of the Teichmüller space, and it is interesting also in our situation. We will give a comment on this in Remark 2 at the end of section 7 .

Acknowledgement. The author wishes to thank K. Akutagawa, O. Kobayashi, T. Nakanishi and S. Nayatani for useful discussions and their interest in this work.

\section{The SPACE OF FLAT CONFORMAL STRUCTURES}

Let $M$ be a paracompact $C^{r}$ manifold, $r \geq 0$. In this section, we assume that $\operatorname{dim} M=n \geq 3$. We define the space of flat conformal structures by means of developing maps associated to $C^{r}$ Möbius structures $(r \geq 0)$. The most important result in this section is a regularity lemma (Lemma 2.4), which is a corollary to the holonomy theorem (Theorem 2.3). Some other useful observations are given in the remarks following Definitions 2.1 and 2.2. For expositions on the subject treated in this section, we refer the reader to [2], [6] and their references.

Definition 2.1. Let $\left\{U_{\lambda}\right\}, \lambda \in \Lambda$, be a collection of open subsets of $M$ and $\varphi_{\lambda}: U_{\lambda} \longrightarrow S^{n} C^{r}$ embeddings into $S^{n}$. If $\left\{\left(U_{\lambda}, \varphi_{\lambda}\right)\right\}_{\lambda \in \Lambda}$ satisfies the following conditions, $\left\{\left(U_{\lambda}, \varphi_{\lambda}\right)\right\}_{\lambda \in \Lambda}$ is called a $C^{r}$ Möbius atlas.

(1) $\left\{U_{\lambda}\right\}_{\lambda \in \Lambda}$ is an open covering of $M$, and $U_{\lambda} \cap U_{\mu}$ is connected if it is nonempty.

(2) If $U_{\lambda} \cap U_{\mu}$ is nonempty, then there is a unique conformal transformation $\psi$ of $S^{n}$ such that $\left.\varphi_{\mu} \circ \varphi_{\lambda}^{-1}\right|_{\varphi_{\lambda}\left(U_{\lambda} \cap U_{\mu}\right)}$ is the restriction of $\psi$.

A maximal Möbius atlas is called a Möbius structure on $M$.

Remark 1. Under our assumption $n \geq 3$, a flat conformal structure on $M$ is nothing but a $C^{\infty}$ Möbius structure on $M$ by Liouville's theorem. On the other hand, given a $C^{\infty}$ Möbius atlas, we can construct a $C^{\infty}$ conformally flat metric by piecing together $\left\{\varphi_{\lambda}^{*} g_{0}\right\}_{\lambda \in \Lambda}$ by a partition of unity subordinate to $\left\{U_{\lambda}\right\}_{\lambda \in \Lambda}$, where $\varphi_{\lambda}^{*} g_{0}$ is the pull-back of the standard metric $g_{0}$ on $S^{n}$ by $\varphi_{\lambda}$.

Remark 2. If $M$ is simply connected, we can construct a $C^{r}$ immersion from $M$ into $S^{n}$ from a given $C^{r}$ Möbius atlas. This follows from a standard monodromy argument. This immersion is called a developing map and it is unique up to composition with a conformal transformation of $S^{n}$. Therefore, if there is a $C^{r}$ Möbius atlas on a manifold $M$, by lifting the Möbius atlas to the universal covering space $\tilde{M}$ of $M$, we can construct a developing map $\Psi$ defined on $\tilde{M}$. We call $\Psi$ also a developing map of $M$. In this case, the fundamental group $\pi_{1}(M)$ of $M$ acts on $\tilde{M}$ as the deck transformation group, and this action preserves the $C^{r}$ Möbius structure on $\tilde{M}$. By the uniqueness of the developing map, $\Psi \circ \gamma=\xi \circ \Psi$ holds for some conformal transformation $\xi$, where $\gamma \in \pi_{1}(M)$. It is easy to see that this gives rise to a representation $\rho: \pi_{1}(M) \longrightarrow \operatorname{Conf}\left(S^{n}\right)$. This representation is called the holonomy representation of $\Psi$.

Remark 3. Let $M$ be a $C^{\infty}$ manifold with a $C^{0}$ Möbius atlas $\left\{\left(U_{\lambda}, \varphi_{\lambda}\right)\right\}_{\lambda \in \Lambda}$. Then, since conformal transformations of $S^{n}$ are smooth, $\left\{\left(U_{\lambda}, \varphi_{\lambda}\right)\right\}_{\lambda \in \Lambda}$ defines a $C^{\infty}$ coordinate system on $M$. However, it is not equivalent to the original $C^{\infty}$ coordinate 
system on $M$ in general. It is not clear whether these two coordinate systems define the same differentiable structure on $M$.

Definition 2.2. Denote by $\operatorname{Diff}_{H}^{r}(M)$ the group consisting of $C^{r}$ diffeomorphisms of $M$ homotopic to the identity. We topologize $\operatorname{Diff}_{H}^{r}(M)$ by the compact- $C^{r}$ topology. We define spaces of flat conformal structures as follows:

$$
\begin{aligned}
& \widetilde{\mathcal{F C}^{r}}(M)=\left\{C^{r} \text { developing maps of } M\right\}, \\
& \mathcal{F C}^{r}(M)=\operatorname{Conf}\left(S^{n}\right) \backslash \widetilde{\mathcal{F C}^{r}}(M), \\
& \mathcal{T}^{r}(M)=\mathcal{F C}^{r}(M) / \operatorname{Diff}_{H}^{r}(M),
\end{aligned}
$$

where the action of $\operatorname{Conf}\left(S^{n}\right)$ on $\widetilde{\mathcal{F C}^{r}}(M)$ is defined by composition on the left. Then $\mathcal{F C}^{r}(M)$ can be viewed as the space of $C^{r}$ Möbius structures. Thus the action of $\eta \in \operatorname{Diff}_{H}^{r}(M)$ on $\mathcal{F C}^{r}(M)$ is defined by the pull-back of a $C^{r}$ Möbius atlas by $\eta$. We topologized the space $\widetilde{\mathcal{F C}^{r}}(M)$ by the compact- $C^{r}$ topology, if $r \geq 1$. We define the topology of $\widetilde{\mathcal{F C}}{ }^{0}(M)$ by means of a subbasis consisting of sets $A, B$ of the following two types.

(1) $A$ is open with respect to the compact open topology of $\widetilde{\mathcal{F C}}(M)$.

(2) For a compact set $K$ of $\tilde{M}, B$ consists of developing maps which are injective on $K$.

The topology of $\mathcal{F C}^{r}(M)$ and $\mathcal{T}^{r}(M)$ is given by the quotient topology. The space $\mathcal{T}^{\infty}(M)$ coincides with $\mathcal{T}(M)$ defined in the introduction.

Remark 1. Let $\widetilde{\operatorname{Diff}}{ }_{H}^{r}(M)$ be the group consisting of lifts of elements in $\operatorname{Diff}_{H}^{r}(M)$ to the universal covering space that commute with all the deck transformations. The topology of this group is given by the compact- $C^{r}$ topology. Clearly, this group $\widetilde{\operatorname{Diff}_{H}^{r}}(M)$ is nonempty and acts continuously on $\widetilde{\mathcal{F C}^{r}}(M)$ by composition on the right. Passing to the quotient, this right action of $\widetilde{\operatorname{Diff}_{H}^{r}}(M)$ gives rise to that of $\operatorname{Diff}_{H}^{r}(M)$ on $\mathcal{F C}^{r}(M)$ mentioned above. Let us consider the map hol : $\widetilde{\mathcal{F C}^{r}}(M) \longrightarrow \operatorname{Hom}\left(\pi_{1}(M), \operatorname{Conf}\left(S^{n}\right)\right)$ that assigns the holonomy representation of $\Psi$ to $\Psi \in \widetilde{\mathcal{F C}^{r}}(M)$. It is clear that this map hol is continuous for any $r$. Here, we define the topology of $\operatorname{Hom}\left(\pi_{1}(M), \operatorname{Conf}\left(S^{n}\right)\right)$ by the pointwise convergence of representations with respect to the topology of $\operatorname{Conf}\left(S^{n}\right)$ as a Lie group. If $\pi_{1}(M)$ is finitely generated, this topology coincides with that given by uniform convergence on the finite set of generators. By definition, the action of $\widetilde{\operatorname{Diff}_{H}^{r}}(M)$ on $\widetilde{\mathcal{F C}^{r}}(M)$ preserves the holonomy representation. In other words, the map hol is constant on each orbit of $\widetilde{\operatorname{Diff}_{H}^{r}}(M)$. Let us define the space $\tilde{\mathcal{T}}^{r}(M)$ as $\widetilde{\mathcal{F C}^{r}}(M) / \widetilde{\operatorname{Diff}_{H}^{r}}(M)$. Then hol induces the map $h o l^{\prime}: \tilde{\mathcal{T}}^{r}(M) \longrightarrow \operatorname{Hom}\left(\pi_{1}(M), \operatorname{Conf}\left(S^{n}\right)\right)$, and this map hol' is clearly continuous. We also note here that $\mathcal{T}^{r}(M)$ is obtained as $\operatorname{Conf}\left(S^{n}\right) \backslash \tilde{\mathcal{T}}^{r}(M)$. We denote the quotient map $\widetilde{\mathcal{F} \mathcal{C}^{r}}(M) \longrightarrow \mathcal{F C}^{r}(M)$ (resp. $\left.\widetilde{\mathcal{F C}^{r}}(M) \longrightarrow \tilde{\mathcal{T}}^{r}(M)\right)$ by $p_{1}$ (resp. $p_{1}^{\prime}$ ). The quotient map from $\mathcal{F C}^{r}(M)$ (resp. $\left.\tilde{\mathcal{T}}^{r}(M)\right)$ is denoted by $p_{2}$ (resp. $\left.p_{2}^{\prime}\right)$. Since each element of $\operatorname{Diff}_{H}^{r}(M), \widetilde{\operatorname{Diff}_{H}^{r}}(M)$ and $\operatorname{Conf}\left(S^{n}\right)$ acts homeomorphically on the corresponding space, the quotient 
maps $p_{1}, p_{2}, p_{1}^{\prime}$ and $p_{2}^{\prime}$ are open. We denote $p_{2} \circ p_{1}=p_{2}^{\prime} \circ p_{1}^{\prime}$ by $\Pi$.

$$
\begin{aligned}
& \widetilde{\mathcal{F C}^{r}}(M) \\
& \operatorname{Conf}\left(S^{n}\right) \backslash \widetilde{\mathcal{F C}}{ }^{r}(M)=\mathcal{F C}^{r}(M) \quad \tilde{\mathcal{T}}^{r}(M)=\widetilde{\mathcal{F C}^{r}}(M) / \widetilde{\operatorname{Diff}_{H}^{r}}(M) \\
& p_{2} \searrow \quad \swarrow p_{2}^{\prime} \\
& \mathcal{F C}^{r}(M) / \operatorname{Diff}_{H}^{r}(M)=\mathcal{T}^{r}(M)=\operatorname{Conf}\left(S^{n}\right) \backslash \tilde{\mathcal{T}}^{r}(M) \\
& \widetilde{\mathcal{F C}^{r}}(M) \quad \text { hol } \\
& \downarrow p_{1}^{\prime} \quad \searrow \\
& \tilde{\mathcal{T}}^{r}(M) \underset{h o l^{\prime}}{\longrightarrow} \operatorname{Hom}\left(\pi_{1}(M), \operatorname{Conf}\left(S^{n}\right)\right)
\end{aligned}
$$

Remark 2. Suppose that $\Psi, \Psi^{\prime} \in \widetilde{\mathcal{F C}^{r}}(M) \subset \widetilde{\mathcal{F C}^{0}}(M)$ define the same element in $\tilde{\mathcal{T}}^{0}(M)=\widetilde{\mathcal{F C}}^{0}(M) / \widetilde{\operatorname{Homeo}}_{H}(M)$, where $\operatorname{Homeo}_{H}(M)=\operatorname{Diff}_{H}^{0}(M)$ is the space of homeomorphisms homotopic to the identity. Then there is a homeomorphism $\eta$ homotopic to the identity such that $\Psi=\Psi^{\prime} \circ \tilde{\eta}$, where $\tilde{\eta}$ is a lift of $\eta$ to the universal cover $\tilde{M}$ of $M$. Since $\Psi$ and $\Psi^{\prime}$ are local $C^{r}$ diffeomorphisms, by taking the composition with $\Psi^{\prime-1}$ locally, we see that $\eta$ must be a $C^{r}$ diffeomorphism. In particular, $\Psi$ and $\Psi^{\prime}$ define the same element in $\tilde{\mathcal{T}}^{r}(M)$. This shows that the natural inclusion of $\widetilde{\mathcal{F C}^{r}}(M)$ into $\widetilde{\mathcal{F C}^{0}}(M)$ induces a continuous injection $\iota: \tilde{\mathcal{T}}^{r}(M) \longrightarrow$ $\tilde{\mathcal{T}}^{0}(M)$. The same is true for $\mathcal{T}^{r}(M)$ and we have a continuous injection $\mathcal{T}^{r}(M) \longrightarrow$ $\mathcal{T}^{0}(M)$.

Remark 3. If $r \geq 1$, the space $\mathcal{F C}^{r}(M)$ is homeomorphic to

$$
\text { \{conformally flat } \left.C^{r-1} \text { Riemannian metrics }\right\} / C_{+}^{r-1}(M)
$$

with the topology induced from the compact- $C^{r-1}$ topology on the space of $C^{r-1}$ Riemannian metrics. Here $C_{+}^{r-1}(M)$ is the space of positive $C^{r-1}$ functions and its action on the space of Riemannian metrics is defined by the multiplication.

Remark 4. The quotient topology on $\mathcal{F C}^{r}(M)$ coincides with the topology defined by the convergence of the coordinate functions of $C^{r}$ Möbius atlases with respect to the compact- $C^{r}$ topology. See [2, section 1.5].

The following theorem, due to Lok, is called the holonomy theorem in the literature and gives us information on the local structure of $\overline{\mathcal{F C}^{r}}(M)$ of a compact manifold $M$.

Theorem 2.3 (see [2] and [6]). Let $\Psi$ be a $C^{r}$ developing map of a compact manifold $M$. Then there is a neighborhood $V \subset \widetilde{\mathcal{F C}^{r}}(M)$ of $\Psi$ homeomorphic to $V_{1} \times V_{2}$, where $V_{1} \subset \operatorname{Hom}\left(\pi_{1}(M), \operatorname{Conf}\left(S^{n}\right)\right)$ is a neighborhood of the holonomy representation of $\Psi$ and $V_{2}$ is a neighborhood of the identity in $\operatorname{Diff}_{H}^{r}(M)$.

Theorem 2.3 asserts that the map hol induces a local homeomorphism

$$
h o l^{\prime}: \tilde{\mathcal{T}}^{r}(M) \longrightarrow \operatorname{Hom}\left(\pi_{1}(M), \operatorname{Conf}\left(S^{n}\right)\right)
$$


for any $r$. When we consider the convergence of certain sequences in $\mathcal{T}^{r}(M)$, Theorem 2.3 can be viewed as a kind of "regularity theorem". For instance, we can prove the following lemma by using Theorem 2.3. Recall that the natural inclusion of $\widetilde{\mathcal{F C}^{r}}(M)$ into $\overline{\mathcal{F C}^{0}}(M)$ induces a continuous injection $\iota: \tilde{\mathcal{T}}^{r}(M) \longrightarrow \tilde{\mathcal{T}}^{0}(M)$ for any $r$.

Lemma 2.4. If $M$ is compact, then $\iota$ is an open map and, in particular, a homeomorphism onto its image. Moreover the image $\iota\left(\tilde{\mathcal{T}}^{r}(M)\right)$ of $\tilde{\mathcal{T}}^{r}(M)$ is open and closed in $\tilde{\mathcal{T}}^{0}(M)$ for any $r$. The same is true for $\mathcal{T}^{r}(M)$.

Proof. Except for the closedness of $\iota\left(\tilde{\mathcal{T}}^{r}(M)\right)$, the assertions in the lemma are straightforward consequences of Theorem 2.3. Therefore we only show the closedness. Take an element $p_{1}^{\prime}(\Psi)$ in the closure of $\iota\left(\tilde{\mathcal{T}}^{r}(M)\right)$. As we have mentioned in Remark 3 following Definition 2.1, $\Psi$ gives a $C^{\infty}$ coordinate system on the underlying topological manifold $N$ of $M$. Denote by $M^{\prime}$ the $C^{\infty}$ manifold homeomorphic to $N$ whose $C^{\infty}$ coordinate system is given by $\Psi$. Since the image of $\tilde{\mathcal{T}}^{\infty}\left(M^{\prime}\right)$ under the injection $\iota^{\prime}: \tilde{\mathcal{T}}^{\infty}\left(M^{\prime}\right) \longrightarrow \tilde{\mathcal{T}}^{0}\left(M^{\prime}\right)=\tilde{\mathcal{T}}^{0}(M)$ induced from the inclusion $\widetilde{\mathcal{F C}}{ }^{\infty}\left(M^{\prime}\right) \longrightarrow \widetilde{\mathcal{F} \mathcal{C}^{0}}\left(M^{\prime}\right)=\widetilde{\mathcal{F C}^{0}}(M)$ is open, $\iota\left(\tilde{\mathcal{T}}^{r}(M)\right) \cap \iota^{\prime}\left(\tilde{\mathcal{T}}^{\infty}\left(M^{\prime}\right)\right)$ is nonempty. Therefore, there are $\Psi_{0} \in \widetilde{\mathcal{F} \mathcal{F}^{r}}(M)$ and $\Psi_{0}^{\prime} \in \widetilde{\mathcal{F} \mathcal{C}^{\infty}}\left(M^{\prime}\right)$ such that $p_{1}^{\prime}\left(\Psi_{0}\right)=p_{1}^{\prime}\left(\Psi_{0}^{\prime}\right)$ in $\tilde{\mathcal{T}}^{0}(N)=\tilde{\mathcal{T}}^{0}(M)=\tilde{\mathcal{T}}^{0}\left(M^{\prime}\right)$. In other words, there is a homeomorphism $\eta$ of $N$, which is homotopic to the identity, satisfying $\Psi_{0}=\Psi_{0}^{\prime} \circ \tilde{\eta}$ for some lift $\tilde{\eta}$ of $\eta$. Composing $\Psi_{0}^{\prime-1}$ locally, we see that this $\eta$ is a $C^{r}$ diffeomorphism between $M$ and $M^{\prime}$. This means $\Psi \circ \tilde{\eta} \in \widetilde{\mathcal{F C}^{r}}(M)$, and hence $p_{1}^{\prime}(\Psi)=p_{1}^{\prime}(\Psi \circ \tilde{\eta}) \in \iota\left(\tilde{\mathcal{T}}^{r}(M)\right)$. Thus $\iota\left(\tilde{\mathcal{T}}^{r}(M)\right)$ is closed in $\tilde{\mathcal{T}}^{0}(M)$.

We will use the closedness part of this lemma in the proof of the main theorem to obtain the regularity of the limit of a certain convergent sequence in $\widetilde{\mathcal{F C}}{ }^{\infty}(M)$.

In the rest of this section, we study the orbits of $\operatorname{Conf}\left(S^{n}\right), \operatorname{Diff}_{H}^{r}(M)$ and $\widetilde{\operatorname{Diff}_{H}^{r}}(M)$ in the corresponding spaces of flat conformal structures. Lemma 2.5 below is easy to verify by using Liouville's theorem.

Lemma 2.5. Let $\Phi \in \widetilde{\mathcal{F C}^{r}}(M)$. The map defined by $\eta \longmapsto \eta \circ \Phi$ is a homeomorphism from $\operatorname{Conf}\left(S^{n}\right)$ onto the $\operatorname{Conf}\left(S^{n}\right)$-orbit of $\Phi$. In particular, it is an open map.

The following lemma is a straightforward corollary to Theorem 2.3 for a compact manifold $M$. However, we will apply it to noncompact manifolds in the case $r=0$. So we give a detailed proof here.

Lemma 2.6. (1) Let $\Psi \in \widetilde{\mathcal{F C}^{r}}(M)$ and $\tilde{\varphi} \in \widetilde{\widetilde{\operatorname{Diff}}_{H}^{r}}(M)$. Then the map defined by $\tilde{\varphi} \mapsto \Psi \circ \tilde{\varphi}$ is a local homeomorphism from $\widetilde{\operatorname{Diff}_{H}^{r}}(M)$ onto the $\widetilde{\operatorname{Diff}_{H}^{r}}(M)$-orbit of $\Psi$.

(2) Suppose that $\mathrm{Homeo}_{H}(M)$ is a closed subgroup of $\operatorname{Homeo}(M)$, and let $\Psi \in$ $\widetilde{\mathcal{F C}^{r}}(M)$. Then the $\widetilde{\operatorname{Diff}_{H}^{r}}(M)$-orbit of $\Psi$ is closed in $\widetilde{\mathcal{F C}^{r}}(M)$.

(3) Let $C \in \mathcal{F C}^{r}(M)$ and $\varphi \in \operatorname{Diff}_{H}^{r}(M)$. Then the map defined by $\varphi \mapsto \varphi^{*} C$ is an open map onto the $\operatorname{Diff}_{H}^{r}(M)$-orbit of $C$.

Proof. (1) Let $G_{\Psi}=\left\{\tilde{\varphi} \in \widetilde{\operatorname{Diff}_{H}^{r}}(M) ; \Psi \circ \tilde{\varphi}=\Psi\right\}$ be the stabilizer of $\Psi$. For any point $x \in \tilde{M}$, there is a neighborhood $U$ of $x$ such that $\Psi$ is injective on $U$. Let $\tilde{\psi} \in G_{\Psi}$. It is easy to see that $\tilde{\psi}(U) \cap U=\emptyset$ unless $\tilde{\psi}=i d_{\tilde{M}}$. Thus, for a 
neighborhood $A=\left\{\tilde{\varphi} \in \widetilde{\operatorname{Diff}_{H}^{r}}(M) ; \tilde{\varphi}(x) \in U\right\}$ of $i d_{\tilde{M}}, \tilde{\psi} \cdot A \cap A=\emptyset$ for any $\tilde{\psi} \in G_{\Psi}, \tilde{\psi} \neq i d_{\tilde{M}}$, where $\tilde{\psi} \cdot A=\{\tilde{\psi} \circ \tilde{\varphi} ; \tilde{\varphi} \in A\}$. Then, it is clear that our

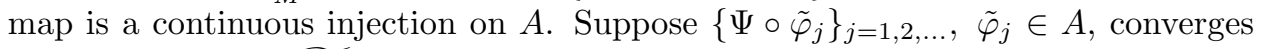
to $\Psi_{\infty}$ in $\Psi \cdot A \subset \widetilde{\mathcal{F C}^{r}}(M)$. Then there exists $\tilde{\varphi}_{\infty} \in A$ such that $\Psi_{\infty}=\Psi \circ \tilde{\varphi}_{\infty}$. We prove that $\left\{\tilde{\varphi}_{j}\right\}$ converges to $\tilde{\varphi}_{\infty}$. Let $\hat{V}_{1}$ be a neighborhood of $x$ such that $\tilde{\varphi}_{\infty}\left(\hat{V}_{1}\right) \subset U$. Since $\tilde{\varphi}_{\infty} \in A$, such a $\hat{V}_{1}$ exists. It is clear that $\Psi_{\infty}$ is injective on $\hat{V}_{1}$. By the definition of the topology of $\widetilde{\mathcal{F C}^{r}}(M)$, we may assume $\Psi \circ \tilde{\varphi}_{j}$ is also injective on $\hat{V}_{1}$. Take a neighborhood $V_{1} \subset \hat{V}_{1}$ of $x$ so that the closure $\bar{V}_{1}$ of $V_{1}$ is contained in $\hat{V}_{1}$. Then, for large $j, \Psi \circ \tilde{\varphi}_{j}\left(V_{1}\right) \subset \Psi_{\infty}\left(\hat{V}_{1}\right)$. Since $\Psi$ is injective on $\tilde{\varphi}_{\infty}\left(\hat{V}_{1}\right) \subset U$, we can compose the inverse $\left(\left.\Psi\right|_{\tilde{\varphi}_{\infty}\left(\hat{V}_{1}\right)}\right)^{-1}$ to $\Psi \circ \tilde{\varphi}_{j}$ on the left. Then $\left(\left.\Psi\right|_{\tilde{\varphi}_{\infty}\left(\hat{V}_{1}\right)}\right)^{-1} \circ\left(\Psi \circ \tilde{\varphi}_{j}\right)$ is the restriction of $\tilde{\varphi} \circ \tilde{\varphi}_{j}$ to $V_{1}$ for some $\tilde{\varphi} \in G_{\Psi}$. But, for large $j, \Psi \circ \tilde{\varphi}_{j}(x)$ must be in $\Phi_{\infty}\left(\hat{V}_{1}\right)$ and hence $\left(\left.\Psi\right|_{\Psi_{\infty}\left(\hat{V}_{1}\right)}\right)^{-1} \circ\left(\Psi \circ \tilde{\varphi}_{j}\right)(x)$ is in $U$. Therefore $\left(\left.\Psi\right|_{\tilde{\varphi}_{\infty}\left(\hat{V}_{1}\right)}\right)^{-1} \circ\left(\Psi \circ \tilde{\varphi}_{j}\right)=\tilde{\varphi}_{j}$ on $V_{1}$. Then, since $\left\{\Psi \circ \tilde{\varphi}_{j}\right\}$ converges to $\Psi_{\infty},\left\{\tilde{\varphi}_{j}\right\}$ converges to $\tilde{\varphi}_{\infty}$ on $V_{1}$. Let $\hat{V}_{2}$ be a precompact open set on which $\Psi_{\infty}$ is injective. Assume $V_{1} \cap \hat{V}_{2}$ is nonempty. We can choose $V_{2} \subset \hat{V}_{2}$ so that $\bar{V}_{2} \subset \hat{V}_{2}$ and $V_{1} \cap V_{2} \neq \emptyset$. Then, for large $j, \Psi \circ \tilde{\varphi}_{j}$ is injective on $\hat{V}_{2}$ and $\Psi \circ \tilde{\varphi}_{j}\left(V_{2}\right) \subset \Psi_{\infty}\left(\hat{V}_{2}\right)$. Since $\Psi_{\infty}=\Psi \circ \tilde{\varphi}_{\infty}$ is injective on $\hat{V}_{2}, \Psi$ is injective on $\tilde{\varphi}_{\infty}\left(\hat{V}_{2}\right)$, and there is the inverse $\left(\left.\Psi\right|_{\tilde{\varphi}_{\infty}\left(\hat{V}_{2}\right)}\right)^{-1}$ of $\Psi$, which is defined on $\Psi_{\infty}\left(\hat{V}_{2}\right)$. Clearly, $\left(\left.\Psi\right|_{\tilde{\varphi}_{\infty}\left(\hat{V}_{2}\right)}\right)^{-1}=\left(\left.\Psi\right|_{\tilde{\varphi}_{\infty}\left(\hat{V}_{1}\right)}\right)^{-1}$ holds on $\Psi_{\infty}\left(\hat{V}_{1} \cap \hat{V}_{2}\right)$; thus, in particular, it holds on $\Psi_{\infty}\left(V_{1} \cap V_{2}\right)$. Therefore $\left(\left.\Psi\right|_{\tilde{\varphi}_{\infty}\left(\hat{V}_{2}\right)}\right)^{-1} \circ\left(\Psi \circ \tilde{\varphi}_{j}\right)=\tilde{\varphi}_{j}$ on $V_{1} \cap V_{2}$, and hence on $V_{2}$. Then, as we have seen above, $\left\{\tilde{\varphi}_{j}\right\}$ converges to $\tilde{\varphi}_{\infty}$ on $V_{2}$, and hence on $V_{1} \cup V_{2}$. Proceeding this way, we see that $\left\{\tilde{\varphi}_{j}\right\}$ converges to $\tilde{\varphi}_{\infty}$ on any compact set. In other words, $\left\{\tilde{\varphi}_{j}\right\}$ converges to $\tilde{\varphi}_{\infty}$ with respect to the compact- $C^{r}$ topology. Thus the inverse defined on $\Psi \cdot A$ of our map is also continuous.

(2) Suppose that a sequence $\left\{\Psi \circ \tilde{\varphi}_{j}\right\}_{j=1,2, \ldots}$ converges to $\Phi$. We show that $\Phi \in \Psi \cdot \widetilde{\operatorname{Diff}_{H}^{r}}(M)$. The proof is given by a certain modification of that of (1).

Let $x$ be a point of $\tilde{M}$ and $U$ be a precompact neighborhood of $x$ on which $\Phi$ is injective. Then, by our definition of the topology of $\overline{\mathcal{F C}^{r}}(M), j \geq j_{1}$ implies that $\Psi \circ \tilde{\varphi}_{j}$ is injective on $U$ for some large number $j_{1}$. We may assume $\Phi(x) \in \Psi \circ \tilde{\varphi}_{j_{1}}(U)$ and $\Phi \circ \tilde{\varphi}_{j}(x) \in \Psi \circ \tilde{\varphi}_{j_{1}}(U)$ for $j \geq j_{1}$. Let $\Psi_{1}=\Psi \circ \tilde{\varphi}_{j_{1}}$ and $\tilde{\varphi}_{j, 1}=\tilde{\varphi}_{j_{1}}^{-1} \circ \tilde{\varphi}_{j}$. Take a neighborhood $\hat{V}_{1} \subset U$ of $x$ so that $\Phi\left(\hat{V}_{1}\right) \subset \Psi_{1}(U)$. Let $V_{1}$ be an open subset of $\hat{V}_{1}$ such that $\bar{V}_{1} \subset \hat{V}_{1}$. Then $\Psi_{1} \circ \tilde{\varphi}_{j, 1}\left(V_{1}\right)$ is contained in $\Phi\left(\hat{V}_{1}\right)$ for large $j$. Also there is the inverse $\left(\left.\Psi_{1}\right|_{U}\right)^{-1}$ of $\left.\Psi_{1}\right|_{U}$, which is defined on $\Phi\left(\hat{V}_{1}\right) \subset \Psi_{1}(U)$. Let $A=\{\tilde{\varphi} \in$ $\left.\widetilde{\operatorname{Difff}_{H}^{r}}(M) ; \tilde{\varphi}(x) \in U\right\}$ as in the proof of (1). Then, as we have seen above, the map $\tilde{\varphi} \mapsto \Psi_{1} \circ \tilde{\varphi}$ defined on $A$ is a homeomorphism. It is easy to see that the image of $A$ is exactly the set given by $D=\Psi_{1} \cdot \widetilde{\operatorname{Diff}_{H}^{r}}(M) \cap\left\{\Psi^{\prime} \in \widehat{\mathcal{F C}^{r}}(M) ; \Psi^{\prime}(x) \in \Psi_{1}(U)\right\}$. Since $\Psi_{1} \circ \tilde{\varphi}_{j, 1} \in D$ for large $j$, we may assume $\tilde{\varphi}_{j, 1}$ to be an element of $A$. Then, as in the proof of (1), by our choice of $\tilde{\varphi}_{j, 1},\left.\left(\left.\Psi\right|_{U}\right)_{1}^{-1} \circ\left(\Psi_{1} \circ \tilde{\varphi}_{j, 1}\right)\right|_{V_{1}}=\left.\tilde{\varphi}_{j, 1}\right|_{V_{1}}$. Since $\left\{\left.\Psi_{1} \circ \tilde{\varphi}_{j, 1}\right|_{V_{1}}\right\}$ converges to $\left.\Phi\right|_{V_{1}}$, it must be equicontinuous and uniformly bounded. Since $\left(\left.\Psi_{1}\right|_{U}\right)^{-1}$ is uniformly continuous on $\Phi\left(\hat{V}_{1}\right) \subset \Psi_{1}(U),\left\{\tilde{\varphi}_{j, 1}\right\}$ is also equicontinuous and, clearly, uniformly bounded. By taking a subsequence, if necessary, $\left\{\tilde{\varphi}_{j, 1}\right\}$ converges uniformly to some $\tilde{\varphi}_{\infty, 1}$ on $V_{1}$. By the uniqueness of the limit, $\Phi$ must coincide with $\Psi_{1} \circ \tilde{\varphi}_{\infty, 1}$ on $V$. Since $\Phi_{1}$ is a $C^{r}$ diffeomorphism on $V_{1}$ and since $\left(\left.\Psi_{1}\right|_{U}\right)^{-1}$ is a $C^{r}$ diffeomorphism on $\Phi\left(\hat{V}_{1}\right), \tilde{\varphi}_{\infty, 1}=\left(\left.\Psi_{1}\right|_{U}\right)^{-1} \circ \Phi$ is also 
a $C^{r}$ diffeomorphism defined on $V_{1}$. We note here that $\left\{\tilde{\varphi}_{j, 1}\right\}$ actually converges to $\tilde{\varphi}_{\infty, 1}$ in $\widetilde{\operatorname{Diff}_{H}^{r}}(M)$, since $\left\{\Psi_{1} \circ \tilde{\varphi}_{j, 1}\right\}$ converges to $\Phi$ in $\widetilde{\mathcal{F} \mathcal{C}^{r}}(M)$.

Let $\left\{W_{i}\right\}_{i=1,2, \ldots}$ be an exhaustion of $\tilde{M}$. We can choose $W_{1}$ to be $V_{1}$. On $W_{1}$, we already have $\tilde{\varphi}_{\infty}=\tilde{\varphi}_{j_{1}} \circ \tilde{\varphi}_{\infty, 1}$ and $\Phi=\Psi \circ \tilde{\varphi}_{\infty}$. For every point $x \in W_{2}$, there is a neighborhood $U_{x}$ of $x$ on which $\Phi$ is injective. Fix $x \in W_{2}$. Then, for large $j, \Psi \circ \tilde{\varphi}_{j}$ is injective on $U_{x}$. As above, for some large $j_{x}$, we may assume $\Phi(x) \in \Psi \circ \tilde{\varphi}_{j_{x}}\left(U_{x}\right)$ and, for $j \geq j_{x}, \Psi \circ \tilde{\varphi}_{j}(x) \in \Psi \circ \tilde{\varphi}_{j_{x}}\left(U_{x}\right)$. Let $\hat{V}_{x}$ be a neighborhood of $x$ such that $\Phi\left(\hat{V}_{x}\right) \subset \Psi \circ \tilde{\varphi}_{j_{x}}\left(U_{x}\right)$. Choose a smaller neighborhood $V_{x}$ of $x$ so that $\bar{V}_{x} \subset \hat{V}_{x}$. We may also assume that $\Psi \circ \tilde{\varphi}_{j}\left(V_{x}\right) \subset \Phi\left(\hat{V}_{x}\right)$ for $j \geq j_{x}$. Choose a finite subset $\left\{V_{1}, \ldots, V_{k_{0}}\right\}$ of $\left\{V_{x} ; x \in W_{2}\right\}$ so that $V_{1}=W_{1}$ and $V_{1} \cup \cdots \cup V_{k_{0}}=W_{2}$. By renumbering the $V_{k}$ 's, we may assume that $V_{1} \cup \cdots \cup V_{k}$ is connected for each $k$. For $V_{2}$, by our construction, there are neighborhoods $U_{2}$ and $\hat{V}_{2}$ of $V_{2}$, and there exists $j_{2}$ such that $\Psi \circ \tilde{\varphi}_{j}$ is injective on $U_{2}$ and that $\Psi \circ \tilde{\varphi}_{j}\left(V_{2}\right) \subset \Phi\left(\hat{V}_{2}\right)$ for any $j \geq j_{2}$. Set $\Psi_{2}=\Psi \circ \tilde{\varphi}_{j_{2}}$ and $\tilde{\varphi}_{j, 2}=\tilde{\varphi}_{j_{2}}^{-1} \circ \tilde{\varphi}_{j}$. Then the inverse $\left(\left.\Psi_{2}\right|_{U_{2}}\right)^{-1}$ is defined on $\Phi\left(\hat{V}_{2}\right) \subset \Psi_{2}\left(U_{2}\right)$ and $\left(\left.\Psi_{2}\right|_{U_{2}}\right)^{-1}=\left(\left.\Psi_{1} \circ \tilde{\varphi}_{j_{1}}^{-1} \circ \tilde{\varphi}_{j_{2}}\right|_{U_{2}}\right)^{-1}$ on $\Psi_{2}\left(V_{1} \cap V_{2}\right)$. As we have seen above, for large $j,\left(\left.\Psi_{1}\right|_{U}\right)^{-1} \circ \Psi_{1} \circ \tilde{\varphi}_{j_{1}}^{-1} \circ \tilde{\varphi}_{j}=\left(\left.\Psi_{1}\right|_{U}\right)^{-1} \circ \Psi_{1} \circ \tilde{\varphi}_{j, 1}=\tilde{\varphi}_{j, 1}$ holds on $V_{1}$. Thus, for large $j$, we have

$$
\begin{aligned}
\left(\left.\Psi_{2}\right|_{U_{2}}\right)^{-1} \circ\left(\Psi_{2} \circ \tilde{\varphi}_{j, 2}\right) & =\left.\left(\Psi_{1} \circ \tilde{\varphi}_{j_{1}}^{-1} \circ \tilde{\varphi}_{j_{2}}\right)\right|_{U_{2}} ^{-1} \circ\left(\Psi_{1} \circ \tilde{\varphi}_{j_{1}}^{-1} \circ \tilde{\varphi}_{j_{2}} \circ \tilde{\varphi}_{j, 2}\right) \\
& =\tilde{\varphi}_{j_{2}}^{-1} \circ \tilde{\varphi}_{j_{1}} \circ\left(\left.\Psi_{1}\right|_{U}\right)^{-1} \circ\left(\Psi_{1} \circ \tilde{\varphi}_{j_{1}}^{-1} \circ \tilde{\varphi}_{j}\right) \\
& =\tilde{\varphi}_{j_{2}}^{-1} \circ \tilde{\varphi}_{j_{1}} \circ \tilde{\varphi}_{j, 1} \\
& =\tilde{\varphi}_{j_{2}}^{-1} \circ \tilde{\varphi}_{j} \\
& =\tilde{\varphi}_{j, 2}
\end{aligned}
$$

on $V_{1} \cap V_{2}$. Thus $\left(\left.\Psi_{2}\right|_{U_{2}}\right)^{-1} \circ\left(\Psi_{2} \circ \tilde{\varphi}_{j, 2}\right)=\tilde{\varphi}_{j, 2}$ on $V_{2}$. As in the preceding paragraph, $\left\{\tilde{\varphi}_{j, 2}\right\}$ converges to a $C^{r}$ diffeomorphism $\tilde{\varphi}_{\infty, 2}$ and $\Phi=\Psi_{2} \circ \tilde{\varphi}_{\infty, 2}$ on $V_{2}$. It is clear that $\tilde{\varphi}_{j_{1}} \circ \tilde{\varphi}_{\infty, 1}=\tilde{\varphi}_{j_{2}} \circ \tilde{\varphi}_{\infty, 2}$ holds on $V_{1} \cap V_{2}$. Therefore we get $\tilde{\varphi}_{\infty}$ satisfying $\Phi=\Psi \circ \tilde{\varphi}_{\infty}$ on $V_{1} \cup V_{2}$.

Repeating this process, we obtain $\tilde{\varphi}_{\infty}$ defined on $W_{2}$ satisfying $\Phi=\Psi \circ \tilde{\varphi}_{\infty}$. It is clear that this map is a $C^{r}$ diffeomorphism defined on $W_{2}$. In this way, we can extend $\tilde{\varphi}_{\infty}$ to a $C^{r}$ diffeomorphism defined on $W_{i}$. Letting $i \rightarrow \infty$, we get a $C^{r}$ diffeomorphism $\tilde{\varphi}_{\infty}$ on $M$. It is clear that $\tilde{\varphi}_{j}$ converges to $\tilde{\varphi}_{\infty}$ with respect to compact-open topology. Since each $\tilde{\varphi}_{j}$ commutes with all deck transformations on $\tilde{M}$, so does the limit $\tilde{\varphi}_{\infty}$. Thus $\tilde{\varphi}_{\infty}$ defines a $C^{r}$ diffeomorphism $\varphi_{\infty}$ on $M$, and $\varphi_{j} \in \operatorname{Diff}_{H}^{r}(M)$, whose lifts are $\tilde{\varphi}_{j}$, converges to $\varphi_{\infty}$. Since $\operatorname{Homeo}_{H}(M)$ is closed, $\varphi_{\infty} \in \operatorname{Diff}_{H}^{r}(M)=\operatorname{Diff}^{r}(M) \cap \operatorname{Homeo}_{H}(M)$, and hence $\tilde{\varphi}_{\infty} \in \widetilde{\operatorname{Diff}_{H}^{r}}(M)$.

(3) Let $C \in \mathcal{F C}^{r}(M)$ and choose $\Psi \in \widehat{\mathcal{F C}^{r}}(M)$ so that $p_{1}(\Psi)$. Observe that the following diagram commutes:

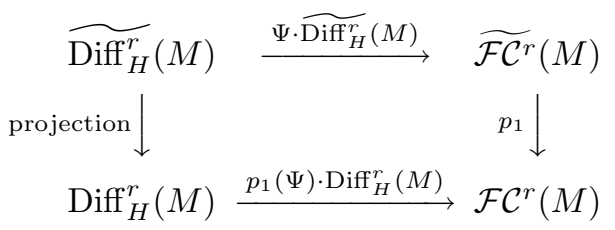

Then, combined with (1), the continuity of the projection $\widetilde{\operatorname{Diff}_{H}^{r}}(M) \longrightarrow \operatorname{Diff}_{H}^{r}(M)$, which is obvious, and the openness of $p_{1}$ imply (3). 
Remark 1. For a compact manifold $M, \operatorname{Homeo}_{H}(M)$ is open and closed in the group $\operatorname{Homeo}(M)$ of homeomorphisms. The same is true for a manifold homeomorphic to the interior of a compact manifold with boundary. These facts easily follow from Corollary 1.1 and 6.1 in [3]. For other manifolds, $\operatorname{Homeo}_{H}(M)$ need not be open. An example can be seen in [3, p. 77]. Though this example is given as a manifold whose group of homeomorphisms is not locally contractible, this is actually an example of such a manifold. However, it is not known, to the author, whether the closedness of $\operatorname{Homeo}_{H}(M)$ is true or not for other manifolds.

Remark 2. The discussion in this section remains valid if we consider the quotient spaces by the action of $\operatorname{Diff}_{C}^{r}(M)$ and $\widetilde{\operatorname{Diff}_{C}^{r}}(M)$ instead of $\operatorname{Diff}_{H}^{r}(M)$ and $\widetilde{\operatorname{Diff}_{H}^{r}}(M)$ respectively. Also we may consider the quotient space by the identity component $\operatorname{Diff}_{0}^{r}(M)$ of $\operatorname{Diff}^{r}(M)$. Here, $\operatorname{Diff}_{C}^{r}(M)$ denotes the subgroup of $\operatorname{Diff}^{r}(M)$ consisting of diffeomorphisms which have a lift to the universal covering space commuting with the deck transformations. This is the largest group of diffeomorphisms that acts on the fundamental group trivially. The group $\widetilde{\operatorname{Diff}_{C}^{r}}(M)$ is defined as the group consisting of the lifts of diffeomorphisms of $M$ which commute with the deck transformations. Clearly, $\operatorname{Diff}_{C}^{r}(M)$ is a closed subgroup of $\operatorname{Diff}^{r}(M)$. Also this group is normal in $\operatorname{Diff}^{r}(M)$, since $\widetilde{\operatorname{Diff}_{C}^{r}}(M)$ is the kernel of the homomorphism $\chi$ from $\widetilde{\operatorname{Diff}^{r}}(M)$ into the group of the automorphisms of the deck transformation group that is defined by conjugation, where $\widetilde{\operatorname{Diff}^{r}}(M)$ is the group of all lifts of the elements of $\operatorname{Diff}^{r}(M)$. The homomorphism $\chi$ is a continuous homomorphism onto its image, where the topology of the image is defined by the pointwise convergence of mappings on a subset of $\operatorname{Diff}^{r}(\tilde{M})$. Since the deck transformation group is a discrete subset of $\operatorname{Diff}^{r}(\tilde{M})$, the image of $\chi$ is totally disconnected with respect to this

topology. Thus $\widetilde{\operatorname{Diff}_{C}^{r}}(M)$ is open in $\widetilde{\operatorname{Diff}^{r}}(M)$. The map $\widetilde{\operatorname{Diff}^{r}}(M) \longrightarrow \operatorname{Diff}^{r}(M)$ is easily seen to be open, and hence $\operatorname{Diff}_{C}^{r}(M)$ is open in $\operatorname{Diff}^{r}(M)$. Obviously, $\operatorname{Diff}_{H}^{r}(M)$ is a subgroup of $\operatorname{Diff}_{C}^{r}(M)$. If $M$ admits a complete metric with nonpositive sectional curvature, then it is easy to see that $\operatorname{Diff}_{C}^{r}(M)$ coincides with $\operatorname{Diff}_{H}^{r}(M)$. A reason to consider the space $\mathcal{T}^{\infty}(M)$ instead of the moduli space is the expectation that $\mathcal{T}^{\infty}(M)$ is less singular than the moduli space, as is true in the case of Riemann surfaces. From this point of view, one of the fundamental questions about the space of flat conformal structures may be to find a reasonable covering (or fiber) space of the moduli space, and there is no reason to restrict our attention to the quotient by $\operatorname{Diff}_{H}^{r}(M)$. In fact, for Riemann surfaces covered by the Euclidean or the hyperbolic plane, the groups $\operatorname{Diff}_{0}^{r}(M), \operatorname{Diff}_{H}^{r}(M)$ and $\operatorname{Diff}_{C}^{r}(M)$ agree. Therefore, the Teichmüller space of such Riemann surfaces can be viewed as the quotient by any of these groups. We cannot give the answer to the question in the present paper, and we mainly consider the quotient by $\operatorname{Diff}_{H}^{r}(M)$, since this is the usual definition of the Teichmüller space of Riemann surfaces.

\section{Quasiconformal mappings and the Teichmüller PSeudodistance}

In this section, we review some basic facts on quasiconformal mappings and define the Teichmüller pseudodistance. We start with the definition of quasiconformal mappings. For expositions on this subject, we refer the reader to [19], [20] and [22]. 
Definition 3.1. Let $\varphi$ be a map between two oriented Riemannian $n$-manifolds $(M, g)$ and $(N, h)$. Suppose that $\varphi$ belongs to $W_{l o c}^{1, n}((M, g),(N, h)) \cap C^{0}(M, N)$. If there is a constant $K \geq 1$ such that $f$ satisfies

$$
\left\|d \varphi_{p}\right\|^{n} \leq K J_{\varphi}(p) \quad \text { a.e., }
$$

then $\varphi$ is called a quasiregular mapping (abbreviated qr mapping), where $\left\|d \varphi_{p}\right\|$ and $J_{\varphi}(p)$ denote the operator norm of the differential map $d \varphi_{p}: T_{p} M \longrightarrow T_{\varphi(p)} N$ and the Jacobian of $\varphi$ respectively. Here, the differential map $d \varphi_{p}$ is a linear map defined by the weak derivatives of $\varphi$. If a qr mapping $\varphi$ is a homeomorphism, then $\varphi$ is called quasiconformal (abbreviated qc).

A nonconstant qr mapping $\varphi$ is open, discrete, orientation preserving and differentiable almost everywhere ([17], see also Chapter VI of [20]).

Definition 3.3. The smallest $K$ satisfying (3.2) is called the outer dilatation of $\varphi$ and is denoted by $K_{O}(\varphi ;(M, g),(N, h))$. If $\varphi$ is qr, then also

$$
J_{\varphi}(p) \leq K^{\prime} \inf _{g(X, X)=1, X \in T_{p} M} h\left(d \varphi_{p}(X), d \varphi_{p}(X)\right)^{n / 2} \quad \text { a.e. }
$$

holds for some $K^{\prime} \geq 1$. The smallest $K^{\prime}$ satisfying (3.4) is called the inner dilatation of $\varphi$ and is denoted by $K_{I}(\varphi ;(M, g),(N, h))$. We call

$$
K(\varphi ;(M, g),(N, h))=\max \left\{K_{O}(\varphi ;(M, g),(N, h)), K_{I}(\varphi ;(M, g),(N, h))\right\}
$$

the maximal dilatation of $\varphi$. If $K(\varphi ;(M, g),(N, h)) \leq K$ for a qr mapping $\varphi$, then $\varphi$ is said to be a $K$-qr mapping.

Let $C_{g}$ and $C_{h}$ be conformal classes containing $g$ and $h$ respectively. Then it is clear that, for a qr mapping $\varphi$ between $(M, g)$ and $(N, h), K_{O}(\varphi ;(M, g),(N, h))=$ $K_{O}\left(\varphi ;\left(M, g^{\prime}\right),\left(N, h^{\prime}\right)\right)$ and $K_{I}(\varphi ;(M, g),(N, h))=K_{I}\left(\varphi ;\left(M, g^{\prime}\right),\left(N, h^{\prime}\right)\right)$ hold for any $g^{\prime} \in C_{g}$ and $h^{\prime} \in C_{h}$. So we also use the notations $K_{O}\left(\varphi ;\left(M, C_{g}\right),\left(N, C_{h}\right)\right)$, $K_{I}\left(\varphi ;\left(M, C_{g}\right),\left(N, C_{h}\right)\right)$ and $K\left(\varphi ;\left(M, C_{g}\right),\left(N, C_{h}\right)\right)$ in what follows. For a discrete, open and orientation preserving map $\varphi$, the quasiregularity of $\varphi$ is characterized by an inequality for the modulus of path families. See, for example, [11]. The following proposition can be proved by using this characterization.

Proposition 3.5. (1) Let $\varphi:\left(M_{1}, g_{1}\right) \longrightarrow\left(M_{2}, g_{2}\right)$ and $\psi:\left(M_{2}, g_{2}\right) \longrightarrow\left(M_{3}, g_{3}\right)$ be qr mappings. Then

$K_{O}\left(\psi \circ \varphi ;\left(M_{1}, g_{1}\right),\left(M_{3}, g_{3}\right)\right) \leq K_{O}\left(\psi ;\left(M_{2}, g_{2}\right),\left(M_{3}, g_{3}\right)\right) K_{O}\left(\varphi ;\left(M_{1}, g_{1}\right),\left(M_{2}, g_{2}\right)\right)$ and

$K_{I}\left(\psi \circ \varphi ;\left(M_{1}, g_{1}\right),\left(M_{3}, g_{3}\right)\right) \leq K_{I}\left(\psi ;\left(M_{2}, g_{2}\right),\left(M_{3}, g_{3}\right)\right) K_{I}\left(\varphi ;\left(M_{1}, g_{1}\right),\left(M_{2}, g_{2}\right)\right)$.

(2) Let $\varphi:(M, g) \longrightarrow(N, h)$ be a qc mapping. Then

$$
K_{O}\left(\varphi^{-1} ;(N, h),(M, g)\right)=K_{I}(\varphi ;(M, g),(N, h))
$$

and

$$
K_{I}\left(\varphi^{-1} ;(N, h),(M, g)\right)=K_{O}(\varphi ;(M, g),(N, h)) .
$$

Let $M$ be a conformally flat manifold (possibly noncompact). We denote by $[C]\left(=p_{2}(C)\right)$ the element of $\mathcal{T}^{\infty}(M)$ represented by $C \in \mathcal{F C}^{\infty}(M)$. Since $\mathcal{T}^{\infty}(M)$ coincides with $\mathcal{T}(M)$ defined in the introduction, we can regard $C$ as a conformal class of conformally flat Riemannian metrics. 
Definition 3.6. The Teichmüller pseudodistance $d$ on $\mathcal{T}(M)$ is defined by

$$
d\left([C],\left[C^{\prime}\right]\right)=\log \inf K\left(\varphi ;(M, C),\left(M, C^{\prime}\right)\right)
$$

for $[C],\left[C^{\prime}\right] \in \mathcal{T}(M)$, where the infimum is taken over all qc mappings homotopic to the identity.

Remark 1. By definition, the dilatations of a qc mapping are measures of the extent that the given mapping fails to be conformal. Thus, $d$ defined above seems to measure the difference of two equivalence classes of flat conformal structures. In fact, by virtue of Proposition 3.5, we see that this defines a pseudodistance on $\mathcal{T}(M)$.

Remark 2. If $M$ is a nonorientable manifold, the definition above does not make sense, since qc mappings are orientation preserving. However, if we replace the condition (3.2) in the definition of the quasiregularity with

$$
\left\|d \varphi_{p}\right\|^{n} \leq K\left|J_{\varphi}(p)\right| \text { a.e. }
$$

then this makes sense for nonorientable manifolds. We define the Teichmüller pseudodistance for nonorientable manifolds by changing the notion of the quasiregularity in this way. In fact, our proof of the main theorem also works for this case.

Remark 3. Recall that a $C^{0}$ Möbius atlas $\left\{\left(U_{\lambda}, \varphi_{\lambda}\right)\right\}_{\lambda \in \Lambda}$ defines a $C^{\infty}$ coordinate system on the underlying topological manifold. Denote this $C^{\infty}$ manifold by $M^{\prime}$. As we have mentioned in Remark 1 following Definition 2.1, we can construct a conformally flat $C^{\infty}$ metric compatible with the flat conformal structure on $M^{\prime}$ defined by $\left\{\left(U_{\lambda}, \varphi_{\lambda}\right)\right\}_{\lambda \in \Lambda}$. Through this fact, we can define the Teichmüller pseudodistance on $\mathcal{T}^{0}(M)$. We also denote this pseudodistance by $d$. By Remark 2 following Definition 2.2, we can regard $\mathcal{T}^{\infty}(M)$ as a subset of $\mathcal{T}^{0}(M)$. It is clear that $d$ on $\mathcal{T}^{\infty}(M)$ is the restriction of $d$ on $\mathcal{T}^{0}(M)$.

Remark 4. By a suitable modification of Definition 3.6, we can define a pseudodistance on the quotient of $\mathcal{F C}^{r}(M)$ by $\operatorname{Diff}_{C}^{r}(M)$ or $\operatorname{Diff}_{0}^{r}(M)$. (For the definition of these groups, see Remark 2 at the end of the preceding section.) Our results on the Teichmüller pseudodistance $d$ in this paper are also true for these pseudodistances. We will mention this precisely at the end of section 6 .

Next, we state some results on qr mappings of $\boldsymbol{R}^{n}$ which we will use in the proof of a certain compactness lemma (Lemma 4.1). For a qr mapping $\varphi$ of $\boldsymbol{R}^{n}$, the dilatations are denoted by $K_{O}(\varphi), K_{I}(\varphi)$ and $K(\varphi)$ for the sake of simplicity.

Theorem 3.7 ([12]). Let $\varphi: B^{n}(0,1) \longrightarrow S^{n}, n \geq 3$, be a locally homeomorphic $K$-qr mapping, where $B^{n}(0, r)$ denotes the Euclidean open $n$-disk with radius $r$. Then $\varphi$ is injective on $B^{n}(0, R)$, where $R=R(n, K)$ is a positive constant depending only on $n$ and $K$.

Theorem 3.8 (see Theorem 1.11 of [20, Chapter III]). Let $G$ be a bounded domain in $\boldsymbol{R}^{n}$ and $\varphi: G \longrightarrow \boldsymbol{R}^{n}$ a bounded qr mapping, and let $F$ be a compact subset of $G$. Set $\alpha=K_{I}(\varphi)^{1 /(1-n)}$ and $C=\lambda_{n} \operatorname{dist}_{\boldsymbol{R}^{n}}(F, \partial G)^{-\alpha} \operatorname{diam}(\varphi(G))$, where $\lambda_{n}$ is a constant depending only on $n$, and $\operatorname{dist}_{\boldsymbol{R}^{n}}$ and diam denote the Euclidean distance between sets and the diameter of a set with respect to the Euclidean distance respectively. Then $\varphi$ satisfies

$$
\operatorname{dist}_{\boldsymbol{R}^{n}}(\varphi(x), \varphi(y)) \leq C \operatorname{dist}_{\boldsymbol{R}^{n}}(x, y)^{\alpha}
$$

for $x \in F$ and $y \in G$. 
Theorem 3.9 (see sections 21 and 37 of [21]). Let $\varphi_{j}: D \longrightarrow \boldsymbol{R}^{n}, j=1,2, \ldots$, be a sequence of $K-q c$ mappings, where $D$ is a domain in $\boldsymbol{R}^{n}$. If $\left\{\varphi_{j}\right\}$ converges uniformly to a nonconstant map $\varphi$, then $\varphi$ is also a K-qc mapping. Moreover, we have

$$
K_{O}(\varphi) \leq \liminf _{j \rightarrow \infty} K_{O}\left(\varphi_{j}\right), \quad K_{I}(\varphi) \leq \liminf _{j \rightarrow \infty} K_{I}\left(\varphi_{j}\right) .
$$

Theorem 3.10 ([5], [18]). Let $\varphi: D \longrightarrow \boldsymbol{R}^{n}$ be a nonconstant 1-qr mapping defined on a domain $D$ in $\boldsymbol{R}^{n}$ with $n \geq 3$. Then $\varphi$ is the restriction of a Möbius transformation.

Theorem 3.10 is known as the generalized Liouville's theorem. Also, it can be considered as a regularity result for qr mappings. A similar regularity result for qc mappings between Riemannian manifolds is proved by Lelong-Ferrand ([9]).

Theorem $3.11([9])$. Nonconstant 1-qc mappings between $C^{\infty}$ Riemannian manifolds are $C^{\infty}$ conformal diffeomorphisms.

Remark. There are results for qr mappings corresponding to Theorems 3.9 and 3.10. See [20], [19] and papers cited there.

\section{A compactness Lemma}

The purpose of this section is to prove a certain compactness lemma (Lemma 4.1) for the bounded sequences with respect to the Teichmüller pseudodistance. In the rest of this paper, we assume that $n \geq 3$.

Suppose that $\left\{\left[C_{j}\right]\right\} \subset \mathcal{T}^{0}(M), j=0,1,2, \ldots$, is a bounded sequence with respect to $d$. In other words, we have a sequence of representatives $\left\{C_{j}\right\} \subset \mathcal{F C}^{0}(M)$, and there is a sequence of qc mappings $\left\{\varphi_{j}\right\}, j=0,1,2, \ldots$, which satisfies $K\left(\varphi_{j} ;\left(M, C_{0}\right),\left(M, C_{j}\right)\right)=K_{j}+\varepsilon_{j}$, where $K_{j}=\exp d\left(\left[C_{0}\right],\left[C_{j}\right]\right)$ and $\lim _{j \rightarrow \infty} \varepsilon_{j}=$ 0 . Also, we may assume that there is a positive constant $K$ such that $K_{j}+\varepsilon_{j} \leq K$ for any $j$. The following lemma asserts the sequential compactness of such sequences in $\mathcal{T}^{0}(M)$. We note that we do not need to assume $M$ to be compact in the following lemma.

Lemma 4.1. There exist a subsequence $\left\{C_{i}\right\}$ of $\left\{C_{j}\right\}$ and $C_{\infty} \in \mathcal{F C}^{0}(M)$ such that $\left\{\varphi_{i}^{*} C_{i}\right\}$ converges to $C_{\infty}$ in $\mathcal{F C}^{0}(M)$. Moreover,

$$
K\left(i d ;\left(M, C_{0}\right),\left(M, C_{\infty}\right)\right) \leq \liminf _{i \rightarrow \infty} K_{i} .
$$

Proof. Since $C_{0}$ is a flat conformal structure, for any point $x$ of $\left(M, C_{0}\right)$, there is a neighborhood $B_{x}$ of $x$ which is conformal to the unit disk of $\boldsymbol{R}^{n}$. We denote the subset of $B_{x}$ which is conformal to the open disk with radius $r$ by $B_{x}(r)$. Set $R=$ $R(n, K)$, where $R(n, K)$ is the positive constant in Theorem 3.7 and $K$ is an upper bound of $K_{j}+\varepsilon_{j}$ as above. Since $M$ admits a Riemannian metric, by considering an exhaustion of $M$, we can choose a countable subset $\left\{B_{\alpha}(R / 3)=B_{x_{\alpha}}(R / 3)\right\}_{\alpha=1,2, \ldots}$ of $\left\{B_{x}(R / 3)\right\}_{x \in M}$ so that this subset forms a locally finite covering of $M$. It is clear that the lift of this open covering to the universal covering $\left(\tilde{M}, \tilde{C}_{0}\right)$ forms a locally finite open covering of $\tilde{M}$ and that each lift of $B_{\alpha}(R / 3)$ is conformally mapped onto a round disk of $S^{n}$ by a developing map of $C_{0}$. Since the intersection of two round disks in $S^{n}$ is connected if it is nonempty, the number of connected components of $B_{\alpha}(R / 3) \cap B_{\beta}(R / 3)$ is finite. Thus, by taking a refinement of this covering, we have a locally finite countable covering $\left\{B_{\alpha}^{\prime}\right\}$ such that $B_{\alpha}^{\prime} \cap B_{\beta}^{\prime}$ is connected if it 
is nonempty. Then, clearly, this defines a $C^{\infty}$ Möbius atlas compatible with $C_{0}$. In what follows, we assume that $B_{\alpha}(R / 3) \cap B_{\beta}(R / 3)$ is either connected or empty for the sake of simplicity.

Let $\left.\varphi_{j} \widetilde{\left(B_{\alpha}(1)\right.}\right)$ be a lift of $\varphi_{j}\left(B_{\alpha}(1)\right)$ to the universal covering space $\tilde{M}$, and let $\Phi_{j}$ be a developing map of $C_{j}$. Denote the map $\Phi_{j} \circ\left(\left.\operatorname{proj}\right|_{\varphi_{j}\left(\widetilde{\left.B_{\alpha}(1)\right)}\right.}\right)^{-1} \circ \varphi_{j}$ from $B_{\alpha}(1)$ into $S^{n}$ by $\psi_{\alpha}^{j}$, where proj is the covering projection.

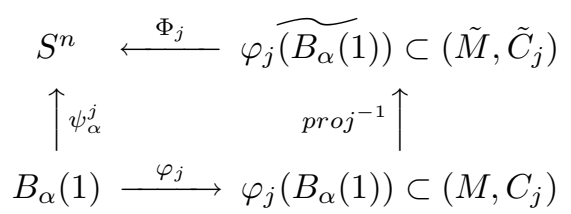

We may regard $\psi_{\alpha}^{j}$ as a $\left(K_{j}+\varepsilon_{j}\right)$-qr local homeomorphism from $B_{\alpha}(1)$ into $S^{n}$, and hence it is injective on $B_{\alpha}(R)$ by Theorem 3.7. Note that $\left\{\left(B_{\alpha}(R / 3), \psi_{\alpha}^{j}\right)\right\}_{\alpha=1,2, \ldots}$ is a Möbius atlas compatible with $\varphi_{j}^{*} C_{j}$. Denote by $B(0, r)$ the disk centered at the origin with radius $r$ in $\boldsymbol{R}^{n}$. By taking composition with an element of $\operatorname{Conf}\left(S^{n}\right)$, we may regard $\psi_{\alpha}^{j}$ restricted to $B_{\alpha}(R)$ as a $K_{j}+\varepsilon_{j}$-qc mapping

$$
\left.\psi_{\alpha}^{j}\right|_{B_{\alpha}}: B(0, R) \longrightarrow B(0,1) .
$$

Here we identify $B_{\alpha}(R)$ (resp. the southern hemisphere of $S^{n}$ ) with $B(0, R)$ (resp. $B(0,1))$. Further, we may assume that the diameter of $\psi_{\alpha}^{j}(B(0, R))$ is equal to 1 for each $\alpha$ and $j$. Consider the sequence $\left\{\left.\psi_{\alpha}^{j}\right|_{B(0,2 R / 3)}\right\}_{j=1,2, \ldots}$ for a fixed $\alpha$. Then this sequence is clearly totally bounded, and also equicontinuous by Theorem 3.8. Thus, by Ascoli's theorem, there is a subsequence $\left\{\left.\psi_{\alpha}^{j(k)}\right|_{B(0,2 R / 3)}\right\}$ which converges uniformly to a continuous map $\psi_{\alpha}^{\infty}$ on $B(0,2 R / 3)$. By Theorem $3.9, \psi_{\alpha}^{\infty}$ is either a constant map or a nonconstant qc mapping. Since the diameter of $\psi_{\alpha}^{j}(B(0, R))$ is equal to 1 for each $j$, the limit $\psi_{\alpha}^{\infty}$ cannot be a constant map. Therefore $\psi_{\alpha}^{\infty}$ is a nonconstant qc mapping defined on $B(0,2 R / 3)$ and, again by Theorem 3.9, $K\left(\psi_{\alpha}^{\infty}\right) \leq \liminf _{k \rightarrow \infty} K_{j(k)}$. By the diagonal argument, there is a subsequence $\{i\}$ of $\{j(k)\}$ such that, for every $\alpha,\left\{\left.\psi_{\alpha}^{i}\right|_{B(0,2 R / 3)}\right\}$ converges uniformly to $\psi_{\alpha}^{\infty}$ on $B(0,2 R / 3)$.

Suppose $B_{\alpha}(R / 3) \cap B_{\beta}(R / 3)$ is nonempty. Let $\tau_{\beta \alpha}^{i}$ be the transition function

$$
\tau_{\beta \alpha}=\psi_{\beta}^{i} \circ\left(\psi_{\alpha}^{i}\right)^{-1}: \psi_{\alpha}^{i}\left(B_{\alpha}(2 R / 3) \cap B_{\beta}(2 R / 3)\right) \longrightarrow \psi_{\beta}^{i}\left(B_{\alpha}(2 R / 3) \cap B_{\beta}(2 R / 3)\right),
$$

for each $i$ and $\infty$. By the preceding paragraph, $\left\{\psi_{\alpha}^{i}\right\}$ converges uniformly to $\psi_{\alpha}^{\infty}$ on $B_{\alpha}(2 R / 3)$, and hence, for large $i, \psi_{\alpha}^{i}\left(B_{\alpha}(2 R / 3) \cap B_{\beta}(2 R / 3)\right)$ contains some neighborhood $U$ of the closure of $\psi_{\alpha}^{\infty}\left(B_{\alpha}(R / 3) \cap B_{\beta}(R / 3)\right)$. Thus $\left(\psi_{\alpha}^{i}\right)^{-1}$ is defined on $U$ for large $i$ and, again by Theorem 3.8 and Ascoli's theorem, we may assume $\left\{\left(\psi_{\alpha}^{i}\right)^{-1}\right\}$ converges uniformly to $\left(\psi_{\alpha}^{\infty}\right)^{-1}$ on $\psi_{\alpha}^{\infty}\left(B_{\alpha}(R / 3) \cap B_{\beta}(R / 3)\right)$. Therefore $\left\{\tau_{\beta \alpha}^{i}\right\}$ converges uniformly to $\tau_{\beta \alpha}^{\infty}$ on $\psi_{\alpha}^{\infty}\left(B_{\alpha}(R / 3) \cap B_{\beta}(R / 3)\right)$. Since $\left\{\left(B_{\alpha}(R / 3), \psi_{\alpha}^{i}\right)\right\}_{\alpha=1,2, \ldots}$ is a Möbius atlas compatible with $\varphi_{i}^{*} C_{i}$, each transition function $\left.\tau_{\beta \alpha}^{i}\right|_{B_{\alpha}(R / 3) \cap B_{\beta}(R / 3)}$ of this atlas is the restriction of a conformal transformation $\eta_{\beta \alpha}^{i}$ of $S^{n}$ for each natural number $i$. Since the sequence of restrictions of the $\eta_{\beta \alpha}^{i}$ 's converges uniformly to a locally defined homeomorphism $\tau_{\beta \alpha}^{\infty},\left\{\eta_{\beta \alpha}^{i}\right\}$ itself converges to a conformal transformation $\eta_{\beta \alpha}^{\infty}$ of $S^{n}$ by Liouville's theorem and by the closedness of $\operatorname{Conf}\left(S^{n}\right)$; thus, $\tau_{\beta \alpha}^{\infty}$ is the restriction of $\eta_{\beta \alpha}^{\infty}$. In particular, $\left\{\left(B_{\alpha}(R / 3), \psi_{\alpha}^{\infty}\right)\right\}_{\alpha=1,2, \ldots}$ is a Möbius atlas on $M$. We denote by $C_{\infty}$ the element of $\mathcal{F C}^{0}(M)$ given by this Möbius atlas. Since each of the coordinate functions and 
the transition functions converges uniformly to that of $\left\{\left(B_{\alpha}(R / 3), \psi_{\alpha}^{\infty}\right)\right\}_{\alpha=1,2, \ldots}$, we can construct a convergent sequence $\left\{\Psi_{i}\right\}$ of developing maps and its limit $\Psi_{\infty}$ in $\widetilde{\mathcal{F C}^{0}}(M)$, where $p_{1}\left(\Psi_{i}\right)=\varphi_{i}^{*} C_{i}$ and $p_{1}\left(\Psi_{\infty}\right)=C_{\infty}$. This shows the first part of Lemma 4.1.

Since each $\psi_{\alpha}^{\infty}: B_{\alpha}(R / 3) \longrightarrow S^{n}$ is $K_{\infty}$-qc, where $K_{\infty}=\liminf _{i \rightarrow \infty} K_{i}, \Psi_{\infty}$ is a $K_{\infty}$-qr mapping between the universal covering of $\left(M, C_{0}\right)$ and $S^{n}$. This implies $(4.2)$.

Remark 1 . We can conclude the convergence of $\left\{\varphi_{i}^{*} C_{i}\right\}$ to $C_{\infty}$ by Remark 4 following Definition 2.2 instead of using the convergence of the developing maps as in the proof above.

Remark 2. By Lemma 4.1, we see that $\left\{\left[C_{i}\right]\right\}$ converges to $\left[C_{\infty}\right]$ in $\mathcal{T}^{0}(M)$ with respect to the original topology of $\mathcal{T}^{0}(M)$. This, however, does not imply the convergence of the sequence with respect to the topology defined by $d$. In general, these two topologies may be quite different. Also, we do not know the uniqueness of the limit of convergent sequences in $\mathcal{T}^{0}(M)$ at the present stage.

\section{The PRoOf of The MAIN THEOREM}

In order to prove that $d$ is a complete distance, we need to examine topological properties of $\mathcal{T}^{0}(M)$. (See Theorem 5.6.) When $M$ is compact, it suffices to prove Corollary 5.3 below, and our aim is nearly achieved by a known result (see Remark following Proposition 5.4). For noncompact manifolds, however, little is known about the topology of $\mathcal{T}^{0}(M)$, and we will study it through the holonomy representation in the next section. Therefore, for the sake of consistency, we give a proof of Corollary 5.3 by means of the holonomy theorem.

We start with a quick review of some fundamental facts about the orbit of $\operatorname{Conf}\left(S^{n}\right)$ in the space $\operatorname{Hom}\left(\pi_{1}(M), \operatorname{Conf}\left(S^{n}\right)\right)$. We refer the reader to [8] for details on this subject. Let $S O(n+1,1)$ be a complex algebraic group defined as the identity component of the isometry group of the quadratic form

$$
q\left(x_{0}, x_{1}, \ldots, x_{n}\right)=-x_{0}^{2}+x_{1}^{2}+\cdots+x_{n}^{2}
$$

in $(n+1)$-dimensional complex linear space $\boldsymbol{C}^{n+1}$. Then the identity component $\operatorname{Conf}_{0}\left(S^{n}\right)$ of $\operatorname{Conf}\left(S^{n}\right)$ is identified with the identity component of the set of real points of $S O(n+1,1)$. For any finitely generated group $G$, $\operatorname{Hom}(G, S O(n+1,1))$ has a natural structure of an affine algebraic variety, and $S O(n+1,1)$ acts algebraically on $\operatorname{Hom}(G, S O(n+1,1))$ by conjugation: $\xi \cdot \rho=\xi^{-1} \circ \rho \circ \xi$ for $\xi \in S O(n+1,1)$ and $\rho \in \operatorname{Hom}(G, S O(n+1,1))$. Then $\operatorname{Hom}\left(G, \operatorname{Conf}_{0}\left(S^{n}\right)\right)$ is an open and closed subset of the set of real points of $\operatorname{Hom}(G, S O(n+1,1))$. In what follows, and throughout this paper, we usually consider $\operatorname{Hom}(G, S O(n+1,1))$ as a topological space with topology given by the pointwise convergence of maps into a Lie group. When we deal with Zariski topology of these spaces, it will be specified such as Zariski open, Zariski closure and so on. Since the action of $S O(n+1,1)$ is algebraic, the orbit $S O(n+1,1) \cdot \rho$ of $\rho \in \operatorname{Hom}(G, S O(n+1,1))$ by this action is open and everywhere dense in its Zariski closure. In particular, its closure coincides with its Zariski closure. The complement of the orbit $S O(n+1,1) \cdot \rho$ in its closure is an algebraic subvariety of strictly lower dimension.

From these facts and results in [23], we easily obtain the following lemma. 
Lemma 5.1. Let $G$ be a finitely generated group.

(1) The $\operatorname{Conf}_{0}\left(S^{n}\right)$-orbit $\operatorname{Conf}_{0}\left(S^{n}\right) \cdot \rho$ of $\rho \in \operatorname{Hom}\left(G, \operatorname{Conf}_{0}\left(S^{n}\right)\right)$ is a connected component of the set of real points of the $S O(n+1,1)$-orbit of $\rho$ in $\operatorname{Hom}(G, S O(n+$ $1,1)$ ).

(2) $\operatorname{Conf}_{0}\left(S^{n}\right) \cdot \rho$ is an algebraic partial manifold in the sense of [23], and homeomorphic to $\operatorname{Conf}_{0}\left(S^{n}\right) / S_{\rho}$, where $S_{\rho}$ is the stabilizer of $\rho$.

(3) The closure of $\operatorname{Conf}_{0}\left(S^{n}\right) \cdot \rho$ is a connected component of a real algebraic variety, and $\operatorname{Conf}_{0}\left(S^{n}\right) \cdot \rho$ is open in its closure. The complement of $\operatorname{Conf}_{0}\left(S^{n}\right) \cdot \rho$ in its closure consists of connected components of a real algebraic subvariety with strictly lower dimension.

Let $\left[C_{0}\right],\left[C_{1}\right] \in \mathcal{T}^{0}(M)$ and suppose that $d\left(\left[C_{0}\right],\left[C_{1}\right]\right)=0$. In other words, suppose that there is a sequence of qc mappings $\left\{\varphi_{j}:\left(M, C_{0}\right) \longrightarrow\left(M, C_{1}\right)\right\}_{j=1,2, \ldots}$ such that $\lim _{j \rightarrow \infty} K_{j}=1$, where $K_{j}$ is the maximal dilatation of $\varphi_{j}$. By setting $C_{j}=C_{1}$ in Lemma 4.1, we see that there is a subsequence of $\left\{\varphi_{j}\right\}$, which we denote by $\left\{\varphi_{i}\right\}$, and $C_{\infty} \in \mathcal{F C}^{0}(M)$ such that $\left\{\varphi_{i}^{*} C_{1}\right\}$ converges to $C_{\infty}$ in $\mathcal{F C}^{0}(M)$. We also see that the identity map is 1 -qc between $\left(M, C_{0}\right)$ and $\left(M, C_{\infty}\right)$ by (4.2). This shows $C_{0}=C_{\infty}$, since a 1-qc mapping is a conformal diffeomorphism by Theorem 3.10 (or Theorem 3.11). More precisely speaking, by our proof of Lemma 4.1, there is a convergent sequence $\left\{\Phi_{i}\right\} \subset \widetilde{\mathcal{F C}^{0}}(M)$ of developing maps and its limit $\Phi_{\infty} \in \widetilde{\mathcal{F C}^{0}}(M)$ such that $p_{1}\left(\Phi_{i}\right)=\varphi_{i}^{*} C_{1}$ and $p_{1}\left(\Phi_{\infty}\right)=C_{\infty}$. If $\Pi\left(\Phi_{i}\right)=\Pi\left(\Phi_{\infty}\right)$ holds in this situation, then this means $\left[C_{1}\right]=\left[C_{\infty}\right]$, and hence $\left[C_{0}\right]=\left[C_{1}\right]$ follows from the discussion above. Therefore, $d$ is a distance on $\mathcal{T}^{0}(M)$. This step is a crucial part in proving that $d$ becomes a distance, and, in this paper, we cannot prove $\Pi\left(\Phi_{i}\right)=\Pi\left(\Phi_{\infty}\right)$ for every manifold. However, by using Lemma 5.1 , we can prove the following.

Lemma 5.2. Suppose that the fundamental group $\pi_{1}(M)$ of $M$ is finitely generated, and that $M$ is orientable. Then hol $\left(\Phi_{\infty}\right)$ is in the $\operatorname{Conf}\left(S^{n}\right)$-orbit $\operatorname{Conf}\left(S^{n}\right) \cdot \operatorname{hol}\left(\Phi_{i}\right)$ of hol $\left(\Phi_{i}\right)$. Moreover, if $M$ is compact, then $\Pi\left(\Phi_{i}\right)=\Pi\left(\Phi_{\infty}\right)$.

Proof. Note that every holonomy representation associated to developing maps of an orientable manifold has its image in $\operatorname{Conf}_{0}\left(S^{n}\right)$. Let $\Phi$ be a developing map of $C_{1}$. Then, by our construction of $\Phi_{i}$ in the proof of Lemma 4.1, $\Phi_{i}=\eta_{i} \circ \Phi \circ \tilde{\varphi}_{i}$ for some $\eta_{i} \in \operatorname{Conf}\left(S^{n}\right)$, where $\tilde{\varphi}_{i}$ is a lift of $\varphi_{i}$. By taking a subsequence and by replacing $\Phi$ and $\eta_{i}$ with $\eta \circ \Phi$ and $\eta_{i} \circ \eta^{-1}$ respectively, where $\eta$ is an orientation reversing element in $\operatorname{Conf}\left(S^{n}\right)$, we may assume the $\eta_{i}$ 's are in $\operatorname{Conf}_{0}\left(S^{n}\right)$. Therefore, $\operatorname{hol}\left(\Phi_{i}\right)=\eta_{i} \cdot \operatorname{hol}(\Phi)$ is in the $\operatorname{Conf}_{0}\left(S^{n}\right)$-orbit $\operatorname{Conf}_{0}\left(S^{n}\right) \cdot \operatorname{hol}(\Phi)$ of $h o l(\Phi)$ for each $i$. By the continuity of the map $h o l, h o l\left(\Phi_{\infty}\right)$ is in the closure of $\operatorname{Conf}_{0}\left(S^{n}\right) \cdot h o l(\Phi)$ in $\operatorname{Hom}\left(\pi_{1}(M), \operatorname{Conf}_{0}\left(S^{n}\right)\right)$. Assume that $h o l\left(\Phi_{\infty}\right)$ is not in $\operatorname{Conf}_{0}\left(S^{n}\right) \cdot h o l(\Phi)$. Let $\Psi$ be a developing map representing $C_{0}$. By Lemma 4.1, $\Pi(\Psi)=\left[C_{0}\right]=\left[C_{\infty}\right]=$ $\Pi\left(\Phi_{\infty}\right)$, as we have seen above. In particular, $\operatorname{hol}\left(\Phi_{\infty}\right) \in \operatorname{Conf}\left(S^{n}\right) \cdot \operatorname{hol}(\Psi)$. Replacing $\Psi$ by $\eta \circ \Psi$ if necessary, where $\eta$ is an orientation reversing element of $\operatorname{Conf}\left(S^{n}\right)$, we may assume $\operatorname{hol}\left(\Phi_{\infty}\right)$ is in $\operatorname{Conf}_{0}\left(S^{n}\right) \cdot \operatorname{hol}(\Psi)$. On the other hand, by considering $\left\{\varphi_{i}^{-1}:\left(M, C_{1}\right) \longrightarrow\left(M, C_{0}\right)\right\}_{1,2, \ldots}$, since $\lim _{i \rightarrow \infty} K\left(\varphi_{i} ;\left(M, C_{1}\right),\left(M, C_{0}\right)\right)=1$, we have a convergent sequence $\left\{\Psi_{i}\right\}$ of developing maps and its limit $\Psi_{\infty}$, where $p_{1}\left(\Psi_{i}\right)=\left(\varphi_{i}^{-1}\right)^{*} C_{0}$ and $\Pi\left(\Psi_{\infty}\right)=\left[C_{1}\right]$ as above. In this case, we may assume $h o l\left(\Psi_{\infty}\right)$ is in the closure of $\operatorname{Conf}_{0}\left(S^{n}\right) \cdot h o l(\Psi)$. Since we have assumed that $\operatorname{hol}\left(\Phi_{\infty}\right)$ is in the complement of $\operatorname{Conf}_{0}\left(S^{n}\right) \cdot \operatorname{hol}(\Phi)$ in its closure, so is $\operatorname{hol}(\Psi)$. The complement itself is a real algebraic subvariety by Lemma 5.1 (3), and hence it 
is closed. Therefore $\operatorname{hol}\left(\Psi_{\infty}\right)$ is in the complement. This contradicts $\Pi\left(\Psi_{\infty}\right)=\left[C_{1}\right]$. This shows the first part of Lemma 5.2.

By Lemma $5.1(2)$, we can identify $\operatorname{Conf}_{0}\left(S^{n}\right) \cdot h o l\left(\Phi_{\infty}\right)$ with $\operatorname{Conf}_{0}\left(S^{n}\right) / S$, where $S$ is the stabilizer of $\operatorname{hol}\left(\Phi_{\infty}\right)$ in $\operatorname{Conf}_{0}\left(S^{n}\right)$. Thus we have a fiber bundle $\operatorname{Conf}_{0}\left(S^{n}\right) \longrightarrow \operatorname{Conf}_{0}\left(S^{n}\right) \cdot h o l\left(\Phi_{\infty}\right)$. Choose a continuous section $s$ defined around $\operatorname{hol}\left(\Phi_{\infty}\right)$ with $s\left(h o l\left(\Phi_{\infty}\right)\right)=i d \in \operatorname{Conf}_{0}\left(S^{n}\right)$. Let $V$ be a neighborhood of $\Phi_{\infty}$ in $\widetilde{\mathcal{F C}^{0}}(M)$ as in Theorem 2.3. By Lemma 2.5, there is a neighborhood $U$ of $i d_{S^{n}} \in \operatorname{Conf}_{0}\left(S^{n}\right)$ such that, for any $\eta \in U, \eta \circ \Phi_{\infty} \in V$. By our choice of $s, \xi_{i}=s\left(h o l\left(\Phi_{i}\right)\right) \in U$ for large $i$. Take such a $\xi_{i}$. Then $\xi_{i} \circ \Phi_{\infty} \in V$ and $\operatorname{hol}\left(\xi_{i} \circ \Phi_{\infty}\right)=\xi_{i} \cdot \operatorname{hol}\left(\Phi_{\infty}\right)=\operatorname{hol}\left(\Phi_{i}\right)$. Thus, by Theorem $2.3, \xi_{i} \circ \Phi_{\infty}=\Phi_{i} \circ \tilde{\varphi}$ for some $\tilde{\varphi} \in \widetilde{\operatorname{Homeo}}_{H}(M)$. Hence $\Pi\left(\Phi_{\infty}\right)=\Pi\left(\Phi_{i}\right)$.

Corollary 5.3. If $M$ is compact (possibly nonorientable), $\Pi\left(\Phi_{\infty}\right)=\Pi\left(\Phi_{i}\right)$.

Proof. We have already seen that this is true for orientable manifolds. Suppose $M$ is nonorientable. Denote its orientable double cover by $\hat{M}$. Let $\widehat{\operatorname{Homeo}}_{H}(M)$ be the group consisting of lifts of elements of $\operatorname{Homeo}_{H}(M)$ to $\hat{M}$ which are homotopic to $i d_{\hat{M}}$ on $\hat{M}$. Then this group is closed in $\operatorname{Homeo}_{H}(\hat{M})$. Let $\Phi_{i}$ and $\Phi_{\infty}$, which are developing maps of $M$, be as above. Then they are also developing maps of $\hat{M}$ which represent lifts of the $C_{i}$ 's and $C_{\infty}$ respectively. Clearly, $\left\{\Phi_{i}\right\}$ converges to $\Phi_{\infty}$ in $\widetilde{\mathcal{F C}^{0}}(\hat{M})$. Let $\xi_{i}$ be as in the last paragraph of the proof of Lemma 5.2 for large $i$. Then, since the $\xi_{i}^{-1} \circ \Phi_{i}$ 's considered as developing maps of $\hat{M}$ have the same holonomy representations, they are in the same $\widetilde{\operatorname{Homeo}}_{H}(\hat{M})$-orbit as we have seen above. Since $\Phi_{\infty}$ is in the closure of the $\widetilde{\operatorname{Homeo}}_{H}(\hat{M})$-orbit of $\xi_{i}^{-1} \circ \Phi_{i}$, Lemma 2.6 (1), (2) and the closedness of $\widehat{\mathrm{Homeo}}_{H}(M)$ show that $\Phi_{\infty}$ actually belongs to the $\widetilde{\operatorname{Homeo}}_{H}(M)$-orbit of $\xi_{i}^{-1} \circ \Phi_{i}$. Therefore $\Pi\left(\Phi_{\infty}\right)=\Pi\left(\Phi_{i}\right)$

Proof of the Main Theorem. By the discussion above, $d$ is a distance on $\mathcal{T}^{0}(M)$ and, in particular, on $\mathcal{T}^{\infty}$. Let $\left\{C_{j}\right\} \subset \mathcal{F} \mathcal{C}^{\infty}(M)$ be a sequence of representatives of a bounded sequence $\left\{\left[C_{j}\right]\right\}$ with respect to $d$. Take a subsequence $\left\{C_{i}\right\}$ and $C_{\infty} \in \mathcal{F C}^{\infty}(M)$ as in Lemma 4.1. Then, by Lemma 4.1, $\iota\left(\left\{\left[C_{i}\right]\right\}\right) \subset \iota\left(\mathcal{T}^{\infty}(M)\right)$ converges to $\left[C_{\infty}\right]$ in $\mathcal{T}^{0}(M)$, where $\iota$ is the map defined in section 2 . Since $\iota$ is a homeomorphism onto its image and $\iota\left(\mathcal{T}^{\infty}(M)\right)$ is closed in $\mathcal{T}^{0}(M)$ by Lemma 2.4, $\left[C_{\infty}\right] \in \mathcal{T}^{\infty}(M)$ and $\left\{\left[C_{i}\right]\right\}$ converges to $\left[C_{\infty}\right]$ in $\mathcal{T}^{\infty}(M)$. It is clear that, for compact $M$, the identity map $\mathcal{T}^{\infty}(M) \longrightarrow\left(\mathcal{T}^{\infty}(M), d\right)$ is continuous, and hence $\left[C_{i}\right] \rightarrow\left[C_{\infty}\right]$ with respect to $d$. Therefore any bounded sequence is compact. This shows that $d$ is complete.

Since $\iota: \mathcal{T}^{\infty}(M) \longrightarrow \mathcal{T}^{0}(M)$ is a homeomorphism, together with the continuity of $\mathcal{T}^{\infty}(M) \longrightarrow\left(\mathcal{T}^{\infty}(M), d\right)$, the identity map $\mathcal{T}^{0}(M) \longrightarrow\left(\mathcal{T}^{0}(M), d\right)$ is continuous. In particular, $\mathcal{T}^{0}(M)$ is Hausdorff. Let $\left\{\left[C_{j}\right]\right\}_{j=1,2, \ldots} \subset \mathcal{T}^{0}(M)$ be a convergent sequence with respect to $d$, and $\left[C_{\infty}\right]$ its limit. By Lemma 4.1 and the Hausdorff property of $\mathcal{T}^{0}(M)$, a subsequence of $\left\{\left[C_{j}\right]\right\}$ converges to a unique element, and it must be $\left[C_{\infty}\right]$ as we have seen above. Therefore $\left[C_{\infty}\right]$ lies in the closure of $\left\{\left[C_{j}\right]\right\}$ with respect to the original topology of $\mathcal{T}^{0}(M)$. Therefore, $\mathcal{T}^{0}(M) \longrightarrow$ $\left(\mathcal{T}^{0}(M), d\right)$ is a bijective closed map, and hence it is an open map. Thus $\mathcal{T}^{0}(M) \longrightarrow$ $\left(\mathcal{T}^{0}(M), d\right)$ is an open and continuous bijection, and hence $\mathcal{T}^{0}(M)$ is homeomorphic to $\left(\mathcal{T}^{0}(M), d\right)$. Combining with Lemma 2.4, we see that $\mathcal{T}^{\infty}(M)$ is homeomorphic to $\left(\mathcal{T}^{\infty}(M), d\right)$. 
In general, we have the following sufficient condition for $d$ to be a distance. Further results will be proved in the next section.

Proposition 5.4. The Teichmüller pseudodistance $d$ is a distance on $\mathcal{T}^{0}(M)$ if $\mathcal{T}^{0}(M)$ satisfies the $T_{0}$-property; namely, for any pair $x, y$ in $\mathcal{T}^{0}(M)$, there is a neighborhood $U$ of either $x$ or $y$ such that $U$ separates $x$ and $y$.

Proof. Suppose $d\left(\left[C_{1}\right],\left[C_{2}\right]\right)=0$ for distinct points $\left[C_{1}\right],\left[C_{2}\right] \in \mathcal{T}^{0}(M)$. We may assume that there is a neighborhood $U$ of $\left[C_{1}\right]$ in $\mathcal{T}^{0}(M)$ such that $\left[C_{2}\right]$ is not in $U$. By the discussion in the paragraph following Lemma 5.1, the closure of $p_{2}^{-1}\left(\left[C_{2}\right]\right)$ in $\mathcal{F C}^{0}(M)$ contains $\left[C_{1}\right]$. Therefore $\left[C_{1}\right]$ must be in the closure of $\left[C_{2}\right]$, which is a subset of the complement of $U$. This is impossible.

Remark. For a compact manifold $M$, it is easy to verify that $\mathcal{T}^{0}(M)$ is Hausdorff from the fact that the moduli space of flat conformal structures on a compact manifold is Hausdorff (see, for example, [7]). Thus we can prove the main theorem by means of this fact. However, the assertion of Corollary 5.3 is much weaker than the $T_{0}$-property of $\mathcal{T}^{0}(M)$, and it is enough for our proof above.

By the following lemma, we see that if $\mathcal{T}^{0}(M)$ satisfies the $T_{0}$-property, then $d$ is actually a complete distance on $\mathcal{T}^{0}(M)$. We state this as Theorem 5.6 below.

Lemma 5.5. If $d$ is a distance on $\mathcal{T}^{0}(M)$, then it is complete. Moreover, the identity map $\left(\mathcal{T}^{0}(M), d\right) \longrightarrow \mathcal{T}^{0}(M)$ is continuous.

Proof. By Lemma 4.1, any Cauchy sequence with respect to $d$ has a subsequence converging to an element of $\mathcal{T}^{0}(M)$ with respect to the original topology of $\mathcal{T}^{0}(M)$. It suffices to show that this subsequence converges to the limit with respect to $d$. Let $\left\{\left[C_{j}\right]\right\}$ be the subsequence and $\left[C_{\infty}\right]$ its limit in $\mathcal{T}^{0}(M)$. Denote by $\left\{\Phi_{j}\right\}$ the corresponding convergent sequence of developing maps that is constructed in the proof of Lemma 4.1, and let $\Phi_{\infty}$ be its limit. Since $\left\{\left[C_{j}\right]\right\}$ is a subsequence of a Cauchy sequence, it is also a Cauchy sequence with respect to $d$. Assume $\left\{\left[C_{j}\right]\right\}$ does not converge to $\left[C_{\infty}\right]$ with respect to $d$. Then there is an $\varepsilon>0$ and a subsequence of $\left\{\left[C_{j}\right]\right\}$, which we denote by $\left\{\left[C_{j}\right]\right\}$ again, such that $\left\{\left[C_{j}\right]\right\}$ lies outside the $\varepsilon$-neighborhood (with respect to $d$ ) of $\left[C_{\infty}\right]$. Since $\left\{\left[C_{j}\right]\right\}$ is also a Cauchy sequence, $\left\{\left[C_{j}\right]\right\}_{j>N}$ is contained in the $\varepsilon / 2$-neighborhood of $\left[C_{N}\right]$ for large $N$. By Lemma 4.1, a closed bounded set with respect to $d$ is sequentially compact in $\mathcal{T}^{0}(M)$, and hence a subsequence of $\left\{\left[C_{j}\right]\right\}$ converges to an element lying in the closure of the $\varepsilon / 2$-neighborhood of $\left[C_{N}\right]$. By our proof of Lemma 4.1, this means that a subsequence of $\left\{\Phi_{j}\right\}$ converges to a developing map different from $\Phi_{\infty}$. Since the space of developing maps is clearly Hausdorff, this is impossible. Thus $\left\{\left[C_{j}\right]\right\}$ actually converges to $\left[C_{\infty}\right]$ with respect to $d$.

By the discussion above, the limit of a convergent sequence with respect to $d$ lies in the closure of the sequence with respect to the original topology of $\mathcal{T}^{0}(M)$. Therefore, as in the proof of the main theorem, the identity map $\left(\mathcal{T}^{0}(M), d\right) \longrightarrow$ $\mathcal{T}^{0}(M)$ is continuous.

Theorem 5.6. If $\mathcal{T}^{0}(M)$ is a $T_{0}$-space, then $d$ is a complete distance on $\mathcal{T}^{0}(M)$. Moreover, the identity map $\left(\mathcal{T}^{0}(M), d\right) \longrightarrow \mathcal{T}^{0}(M)$ is continuous.

Remark 1. If $d$ is not a distance, then the identity map $\left(\mathcal{T}^{0}(M), d\right) \longrightarrow \mathcal{T}^{0}(M)$ may not be continuous. We can say only that one of the limits of a convergent sequence with respect to $d$ lies in the closure of the sequence with respect to the original topology of $\mathcal{T}^{0}(M)$. 
Remark 2. We give here a comment on the comparison of our proof and that for the case of Riemann surfaces. First, we give a rough sketch of the proof for the case of Riemann surfaces along the line of our proof. We have considered qc mappings between $\left(M, C_{0}\right)$ and $\left(M, C_{j}\right)$ in the preceding section. If $M$ is a compact and oriented surface with genus greater than one, then these qc mappings are lifted to the universal coverings which are conformal to the unit disk $B^{2}(0,1)$ of $\boldsymbol{R}^{2}$ for all $j$. Thus the claim corresponding to Lemma 4.1 is reduced to the problem on the compactness of $K$-qc mappings on $B^{2}(0,1)$. After suitable normalization of lifts of the qc mappings $\varphi_{j}$, one sees that the set $\left\{\hat{\varphi}_{j}\right\}$ of normalized mappings is precompact with respect to compact-open topology; there is a subsequence of $\left\{\hat{\varphi}_{j}\right\}$ and a nonconstant qc mapping $\varphi$ that is the limit of the subsequence. (This follows from, for example, a theorem of Mori [15].) As a corollary to this, we get the compactness of $K$-qc mappings between $\left(M, C_{0}\right)$ and $\left(M, C_{1}\right)$ for fixed $C_{1}$. This implies that the Teichmüller pseudodistance is actually a distance. Since $\left(M, C_{j}\right)$ is conformal to $B^{2}(0,1) / \hat{\varphi}_{j} \Gamma \hat{\varphi}_{j}^{-1}$ and the $\hat{\varphi}_{j} \Gamma \hat{\varphi}_{j}^{-1}$ 's are conformal groups acting on $B^{2}(0,1)$, where $\Gamma$ is a discrete conformal group such that $\left(M, C_{0}\right)$ is conformal to $B^{2}(0,1) / \Gamma, \varphi \Gamma \varphi^{-1}$ is also a discrete conformal group acting on $B^{2}(0,1)$. Thus the conformal structure given by $B^{2}(0,1) / \varphi \Gamma \varphi^{-1}$ is the limit of a subsequence of $\left\{\left[C_{j}\right]\right\}$. Since the convergence of a sequence of conformal transformations with respect to compact-open topology implies that with respect to compact- $C^{\infty}$ topology, $\left\{\left[C_{j}\right]\right\}$ actually converges to the limit in $\mathcal{T}(M)$ defined in the introduction. This can be proved, for example, by an argument similar to the proof of the holonomy theorem. As a matter of fact, it is the simplest case that the framework of the holonomy theorem can be applied. See [2] and [6] for the holonomy theorem in the general setting. The completeness of the Teichmüller distance follows easily from the discussion above.

On the other hand, when $n \geq 3$ the universal coverings $\left(\tilde{M}, \tilde{C}_{j}\right)$ are no longer conformal to each other, and the argument above fails. However, through Liouville's theorem, which holds only for the case $n \geq 3$, we can consider the flat conformal structure as the Möbius structure. This allows us, together with Theorem 3.7, to localize the problem as we have done in the preceding section; in order to prove Lemma 4.1, we have had only to find the limit of coordinate functions by virtue of Liouville's theorem and Theorem 3.7. The existence of the limit has been proven by using Theorem 3.8 instead of Mori's theorem in the case of the compactness of qc mappings on $B^{2}(0,1)$ mentioned above. (We note that Theorem 3.7 holds only for the case $n \geq 3$.) Unfortunately, this is not sufficient to prove the main theorem. We have needed to examine the topology of $\mathcal{T}^{0}(M)$ carefully and prove the regularity of the limit as we have seen, and this has been carried out by means of the holonomy theorem.

\section{FuRTHER RESUlTS FOR NONCOMPACT MANIFOLDS}

As we have seen, $d$ is a complete distance on $\mathcal{T}^{0}(M)$ if $\mathcal{T}^{0}(M)$ satisfies the $T_{0^{-}}$ property. Thus $d$ is a complete distance if $\mathcal{T}^{0}(M)$ satisfies the $T_{1}$-property; namely, every point in $\mathcal{T}^{0}(M)$ is closed. In this section, since the $T_{1}$-property is rather easier to check in our case, we consider a sufficient condition for a point in $\mathcal{T}^{0}(M)$ to be closed. We show that $d$ is a complete distance on a subset of $\mathcal{T}^{0}(M)$ for a certain noncompact manifold $M$. This set is open and closed in $\mathcal{T}^{0}(M)$ with respect to the topology defined by $d$. 
Recall that $\operatorname{Conf}\left(S^{n}\right)$ can be identified with the isometry group of the hyperbolic space $\left(B^{n+1}\right.$, Poincaré metric) by extending the action of the isometry to the boundary $S^{n}=\partial B^{n+1}$. Through this identification, we can consider the action of subgroups of $\operatorname{Conf}\left(S^{n}\right)$ on $B^{n+1}$. Let $G$ be a subgroup of $\operatorname{Conf}\left(S^{n}\right)$. We do not assume $G$ to be discrete nor finitely generated. The limit set of $G$, denoted by $\Lambda(G)$, is the set of accumulation points in $S^{n}$ of the $G$-orbit of a point $x \in B^{n+1}$. In the proof of Lemma 6.1 below, we frequently use results stated in $[13, \S 5]$.

Lemma 6.1. Let $\rho=\operatorname{hol}(\Phi)$. Assume that $\operatorname{Homeo}_{H}(M)$ is a closed subgroup of Homeo $(M)$. Unless $\rho\left(\pi_{1}(M)\right)$ itself or any index two subgroup of $\rho\left(\pi_{1}(M)\right)$ has common fixed points in $S^{n} \cup B^{n+1}, \Pi(\Phi)$ is closed in $\mathcal{T}^{0}(M)$.

Proof. Let $\left\{\eta_{j}\right\} \subset \operatorname{Conf}\left(S^{n}\right)$ and $\left\{\tilde{\varphi}_{j}\right\} \in \widetilde{\operatorname{Homeo}}_{H}(M)$. Suppose $\eta_{j} \circ \Phi \circ \tilde{\varphi}_{j}$ converges to $\Phi_{\infty}$ in $\widetilde{\mathcal{F C}^{0}}(M)$. We have to show $\Phi_{\infty} \in \Pi^{-1} \circ \Pi(\Phi)$. If a subsequence of $\left\{\eta_{j}\right\}$ converges to some $\eta_{\infty} \in \operatorname{Conf}\left(S^{n}\right)$, then the corresponding subsequence of $\left\{\Phi \circ \tilde{\varphi}_{j}\right\}$ converges to $\eta_{\infty}^{-1} \circ \Phi_{\infty}$. In this case, $\Phi_{\infty} \in \Pi^{-1} \circ \Pi(\Phi)$ follows from Lemma 2.6 (2). In what follows, we prove the existence of a convergent subsequence of $\left\{\eta_{j}\right\}$. To prove this, we first show that, under our assumption, $\rho\left(\pi_{1}(M)\right)$ contains a free subgroup of rank two.

Let $G=\rho\left(\pi_{1}(M)\right)$. Since $G$ has no common fixed points in $S^{n} \cup B^{n+1}, \Lambda(G)$ consists of more than one point. If $\Lambda(G)$ consists of exactly two points, there is a subgroup of index two in $G$ which fixes each point of $\Lambda(G)$. Thus, by our assumption, $\Lambda(G)$ contains at least three points, and hence $\Lambda(G)$ is an infinite set. By [13, Proposition 5.11], for any two points $x, y \in \Lambda(G)$, there is a loxodromic element in $G$ whose fixed points are arbitrarily near $x, y$. Choose four distinct points of $\Lambda(G)$. Then we can find two loxodromic elements $\xi_{1}, \xi_{2}$ in $G$ so that they have no common fixed points. The group $F$ generated by $\xi_{1}^{k}$ and $\xi_{2}^{l}$ for large $k, l$ is easily seen to be a discrete free subgroup of $G$. (Actually, $F$ is a Schottky group of rank two.) We may assume that $F$ lies in the identity component $\operatorname{Conf}_{0}\left(S^{n}\right)$ of $\operatorname{Conf}\left(S^{n}\right)$ by choosing $k$ and $l$ suitably.

Set $\rho_{j}=\operatorname{hol}\left(\eta_{j} \circ \Phi \circ \tilde{\varphi}_{j}\right)=\eta_{j} \cdot \rho$ and $\rho_{\infty}=h o l\left(\Phi_{\infty}\right)$. By the continuity of the map hol, $\left\{\rho_{j}\right\}$ converges to $\rho_{\infty}$. By taking a subsequence of $\{j\}$ and by replacing $\Phi$ and $\eta_{j}$ with $\eta \circ \Phi$ and $\eta_{j} \circ \eta^{-1}, \eta \in \operatorname{Conf}\left(S^{n}\right) \backslash \operatorname{Conf}_{0}\left(S^{n}\right)$, if necessary, we may assume $\eta_{j} \in \operatorname{Conf}_{0}\left(S^{n}\right)$ for each $j$. Then $\left.\rho_{j} \circ \rho^{-1}\right|_{F}$ can be considered as representations of the free group of rank two into $\operatorname{Conf}_{0}\left(S^{n}\right)$. Note that $\left.\rho_{\infty} \circ \rho^{-1}\right|_{F}$ also defines a representation. Denote these representations by $\hat{\rho}_{j}$ and $\hat{\rho}_{\infty}$ respectively. Clearly, $\left\{\hat{\rho}_{j}\right\}$ converges to $\hat{\rho}_{\infty}$. By [14, Corollary 1.2] and [1, Proposition 2.3], the $\operatorname{Conf}_{0}\left(S^{n}\right)$-orbit of $\hat{\rho}$ is closed in $\operatorname{Hom}\left(F, \operatorname{Conf}\left(S^{n}\right)\right)$. Thus $\hat{\rho}_{\infty}=\theta \cdot \hat{\rho}$ for some $\theta \in \operatorname{Conf}_{0}\left(S^{n}\right)$. The orbit $\operatorname{Conf}_{0}\left(S^{n}\right) \cdot \hat{\rho}$ is homeomorphic to $\operatorname{Conf}_{0}\left(S^{n}\right) / S_{\hat{\rho}}$ as we have seen in the preceding section.

Next, we prove the compactness of the stabilizer $S_{\hat{\rho}}$. Note that neither $F$ nor any finite index subgroup of $F$ have common fixed points in $S^{n} \cup B^{n+1}$. Suppose that $S_{\hat{\rho}}$ is noncompact and has common fixed points in $S^{n}$. Then the number of the fixed points is less than three. Otherwise $S_{\hat{\rho}}$ must be compact. (Note that $S_{\hat{\rho}}$ is a closed subgroup of $\operatorname{Conf}\left(S^{n}\right)$.) Assume that $S_{\hat{\rho}}$ fixes exactly one point, say $x$. Then, for any $\eta \in F$ and $g \in S_{\hat{\rho}}, g \circ \eta(x)=\eta \circ g(x)=\eta(x)$, and hence $\eta(x)$ is a fixed point of $g$. Thus $\eta(x)=x$. Therefore $F$ has a common fixed point $x$, a contradiction. If $S_{\hat{\rho}}$ has two fixed points, then a similar argument shows that $F$ itself or some subgroup of $F$ with index two fixes the fixed points of $S_{\hat{\rho}}$. On the other hand, since we assume $S_{\hat{\rho}}$ to be noncompact, $S_{\hat{\rho}}$ fixes no points in $B^{n+1}$. 
Thus, if $S_{\hat{\rho}}$ has no common fixed points in $S^{n}$, then $S_{\hat{\rho}}$ fixes no points in $S^{n} \cup B^{n+1}$. In this case, $S_{\hat{\rho}}$ contains a loxodromic element $g$ by [13, Corollary 5.14]. Since every element of $F$ commutes with $g, F$ itself or some subgroup of $F$ with index two fixes the fixed points of $g$ as we have seen above. Therefore $S_{\hat{\rho}}$ is compact, and hence $S_{\rho}$, which is a closed subgroup of $S_{\hat{\rho}}$, is also compact.

Identifying the $\operatorname{Conf}_{0}\left(S^{n}\right)$-orbit $\operatorname{Conf}_{0}\left(S^{n}\right) \cdot \hat{\rho}_{\infty}$ of $\hat{\rho}_{\infty}$ with $\operatorname{Conf}_{0}\left(S^{n}\right) / S_{\hat{\rho}_{\infty}}$ by the homeomorphism as mentioned above, we get the fiber bundle $\operatorname{Conf}_{0}\left(S^{n}\right) \longrightarrow$ $\operatorname{Conf}_{0}\left(S^{n}\right) \cdot \hat{\rho}_{\infty}$ whose fiber is $S_{\hat{\rho}_{\infty}}$. Let $s$ be a continuous section defined near $\hat{\rho}_{\infty}$. Then, since $\hat{\rho}_{j}=\left(\eta_{j} \circ \theta^{-1}\right) \cdot \hat{\rho}_{\infty}, \eta_{j} \circ \theta^{-1}=s\left(\hat{\rho}_{j}\right) \circ \eta_{j}^{\prime}$ for some $\eta_{j}^{\prime} \in S_{\hat{\rho}_{\infty}}$. By the compactness of $S_{\hat{\rho}_{\infty}}$, there is a convergent subsequence of $\left\{\eta_{j}^{\prime}\right\}$ and its limit $\eta_{\infty}^{\prime}$. Since the section $s$ is continuous, this shows that $\left\{\eta_{j}\right\}$ itself contains a convergent subsequence.

Let $\mathrm{QC}_{H}^{K}(M, C)$ be a set of $K$-qc transformations on $(M, C)$ homotopic to the identity:

$$
\mathrm{QC}_{H}^{K}(M, C)=\left\{\varphi \in \operatorname{Homeo}_{H}(M) ; K(\varphi ;(M, C),(M, C)) \leq K\right\} .
$$

Lemma 6.2. Let $C \in \mathcal{F C}^{0}(M)$ and suppose that $[C]=p_{2}(C)$ is closed in $\mathcal{T}^{0}(M)$. If $\operatorname{Conf}_{H}(M, C)$ is compact, then $\mathrm{QC}_{H}^{K}(M, C)$ is compact for any $K$.

Proof. Let $\left\{\psi_{j}\right\}$ be a sequence in $\mathrm{QC}_{H}^{K}(M, C)$. Then, by Lemma 4.1, there is a convergent subsequence of $\left\{\psi_{j}^{*} C\right\}$ and its limit $C_{\infty} \in \mathcal{F C}^{0}(M)$. Since $[C]$ is closed in $\mathcal{T}^{0}(M),\left[C_{\infty}\right]=[C]$. Thus $C_{\infty}=\psi_{\infty}^{*} C$ for some $\psi_{\infty} \in \operatorname{Homeo}_{H}(M)$. By Lemma $2.6(3)$, we can choose a sequence $\left\{\varphi_{j}\right\} \subset \operatorname{Homeo}_{H}(M)$ so that $\varphi_{j}^{*} C_{\infty}=$ $\psi_{j}^{*} C=\left(\psi_{\infty}^{-1} \circ \psi_{j}\right)^{*} C_{\infty}$ and $\varphi_{j} \rightarrow i d_{M}$. Then, $\psi_{\infty}^{-1} \circ \psi_{j}=\phi_{j} \circ \varphi_{j}$ for some $\phi_{j} \in \operatorname{Conf}_{H}\left(M, C_{\infty}\right)$. Since $\operatorname{Conf}_{H}\left(M, C_{\infty}\right)$ is compact as well as $\operatorname{Conf}_{H}(M, C)$, there is a convergent subsequence of $\left\{\phi_{j}\right\}$, which we denote by $\left\{\phi_{j}\right\}$ again, and its limit $\phi_{\infty}$. Therefore, by our choice of $\varphi_{j}$, the corresponding subsequence $\left\{\psi_{j}\right\}$ converges to $\psi_{\infty} \circ \phi_{\infty}$. Since the limit of a $K$-qc mapping is also $K$-qc, we obtain the desired result.

Theorem 6.3. Suppose that $[C]$ is a closed point of $\mathcal{T}^{0}(M)$ and that $\operatorname{Conf}_{H}(M)$ is compact. Denote by $\mathcal{T}_{0}^{0}(M)$ the subset of $\mathcal{T}^{0}(M)$ containing $[C]$ defined as

$$
\mathcal{T}_{0}^{0}(M)=\left\{\left[C^{\prime}\right] \in \mathcal{T}^{0}(M) ; d\left([C],\left[C^{\prime}\right]\right)<\infty\right\}
$$

Then $d$ is a complete distance on $\mathcal{T}_{0}^{0}(M)$. Moreover, for any $\left[C^{\prime}\right] \in \mathcal{T}_{0}^{0}(M)$, $\operatorname{Conf}_{H}\left(M, C^{\prime}\right)$ is compact.

Proof. Suppose $d\left(\left[C_{1}\right],\left[C_{2}\right]\right)=0$ for $\left[C_{1}\right],\left[C_{2}\right] \in \mathcal{T}_{0}^{0}(M)$. That is, there exists a sequence $\left\{\varphi_{j}\right\}_{j=1,2, \ldots}$ of qc mappings from $\left(M, C_{1}\right)$ to $\left(M, C_{2}\right)$ such that the $\operatorname{limit}_{j \rightarrow \infty} K\left(\lim _{j} ;\left(M, C_{1}\right),\left(M, C_{2}\right)\right)$ of their maximal dilatations equals 1 . Fix qc mappings $\psi_{1}:(M, C) \longrightarrow\left(M, C_{1}\right)$ and $\psi_{2}:(M, C) \longrightarrow\left(M, C_{2}\right)$. Then $\psi_{2}^{-1} \circ \varphi_{j} \circ \psi_{1}$ are contained in $\mathrm{QC}_{H}^{K}(M, C)$ for some $K$. Taking a subsequence, we may assume $\psi_{2}^{-1} \circ \varphi_{j} \circ \psi_{1}$ converges to $\psi_{\infty}$ with respect to the compact-open topology by Lemma 6.2. Therefore $\varphi_{j}$ converges to $\varphi_{\infty}=\psi_{2} \circ \psi_{\infty} \circ \psi_{1}^{-1}$, and this is a 1-qc (therefore smooth conformal) mapping between $\left(M, C_{1}\right)$ and $\left(M, C_{2}\right)$. Thus $d$ is a distance and it is complete by Lemma 5.5 .

If we replace $C_{2}$ with $C_{1}$ and choose $\varphi_{j}$ to be conformal transformations of $\left(M, C_{1}\right)$, we obtain the compactness of $\operatorname{Conf}_{H}\left(M, C_{1}\right)$. 
Since $\mathcal{T}^{\infty}(M)$ can be considered as a subset of $\mathcal{T}^{0}(M)$ as we have seen in section $2, d$ is a distance on $\mathcal{T}_{0}^{\infty}(M)=\mathcal{T}_{0}^{0}(M) \cap \mathcal{T}^{\infty}(M)$ (it may be incomplete). Note that we have not used the flatness of the conformal structures $C_{1}$ and $C_{2}$ in the proof of Theorem 6.3 except the use of Lemma 5.5. Let $\mathcal{C}(M)$ be the space of conformal structures (not necessarily flat) defined by

$$
\mathcal{C}(M)=\left\{C^{\infty} \text { conformal structures on } M\right\} / \operatorname{Diff}_{H}^{\infty}(M) .
$$

It is clear that we can define a pseudodistance on this space, denoted also by $d$, in the same way as in Definition 3.6. By the proof of Theorem 6.3, we have the following result on $(\mathcal{C}(M), d)$.

Corollary 6.4. Let $M$ be a manifold admitting flat conformal structures. Suppose that there is a flat conformal structure $C$ such that $[C]$ is a closed point of $\mathcal{T}^{0}(M)$ and that $\operatorname{Conf}_{H}(M, C)$ is compact. Denote by $\mathcal{C}_{0}(M)$ the subset of $\mathcal{C}(M)$ containing $[C]$ defined as

$$
\mathcal{C}_{0}(M)=\left\{\left[C^{\prime}\right] \in \mathcal{C}(M) ; d\left([C],\left[C^{\prime}\right]\right)<\infty\right\}
$$

Then $d$ is a distance on $\mathcal{C}_{0}(M)$. Moreover, for any $\left[C^{\prime}\right] \in \mathcal{C}_{0}(M), \operatorname{Conf}_{H}\left(M, C^{\prime}\right)$ is compact.

Remark. In general, even if $\operatorname{Conf}_{H}(M, C)$ is compact, $\mathrm{QC}_{H}^{K}(M, C)$ may be noncompact for some $K$. We give an example here. Let $C$ be a conformal structure on $S^{n}$ different from the standard one. Then $i d_{M}:\left(S^{n}, C\right) \longrightarrow\left(S^{n}, C_{0}\right)$ is $K$-qc for some $K$, where $C_{0}$ is the standard conformal structure on $S^{n}$. It is clear that $\mathrm{QC}_{H}^{2 K}\left(S^{n}, C\right)$ contains $\operatorname{Conf}_{H}\left(S^{n} C_{0}\right)$ as a closed subset, and hence $\mathrm{QC}_{H}^{2 K}\left(S^{n} C\right)$ is noncompact.

There is a distinguished class of flat conformal structures called Kleinian structures (or uniformized flat conformal structures) arising from Kleinian groups. Let $\Gamma$ be a Kleinian group. Choose an open subset $\Omega$ of $S^{n}$ so that $\Gamma$ leaves $\Omega$ invariant and that $\Gamma$ acts freely and properly discontinuously on $\Omega$. Then $\Omega / \Gamma$ is a manifold and it carries a natural flat conformal structure coming from the standard conformal structure on $S^{n}$. By the discussion above, we have the following theorem for such manifolds.

Theorem 6.5. Let $(M, C) \cong \Omega / \Gamma$ be a Kleinian manifold. Suppose that $\Gamma$ is nonelementary and that $\operatorname{Homeo}_{H}(M)$ is closed. Then, on $\mathcal{T}_{0}^{0}(M)$ containing $[C]$, $d$ is a complete distance.

Proof. In this case, $\Gamma$ is the image of the holonomy representation associated to a developing map representing $C$. Since $\Gamma$ is nonelementary, neither $\Gamma$ nor any finite index subgroup of $\Gamma$ has common fixed points in $S^{n} \cup B^{n+1}$. Thus, by Lemma 6.1, $[C]$ is closed in $\mathcal{T}^{0}(M)$. Also, the stabilizer $S$ of the holonomy representation, which is the centralizer of $\Gamma$ in this case, is compact by an argument in the proof of Lemma 6.1. Denote by $\widehat{\operatorname{Conf}_{H}}(M, C)$ the group consisting of lifts of elements of $\operatorname{Conf}_{H}(M, C)$ to $\Omega$ which commute with $\Gamma$. Then this group can be considered as a closed subgroup of $S$ by Liouville's theorem, and hence it is compact. Since there is a continuous surjection $\widehat{\operatorname{Conf}_{H}}(M, C) \longrightarrow \operatorname{Conf}_{H}(M, C)$ induced from the covering projection, $\operatorname{Conf}_{H}(M, C)$ is compact. Then the theorem follows from Theorem 6.3. 
Remark 1. Lemma 6.1 gives a sufficient condition for a point of $\mathcal{T}^{0}(M)$ to be closed. On the other hand, for some $M$, there is a point of $\mathcal{T}^{0}(M)$ which is not closed. We give an example here. Let $M=\boldsymbol{R}^{n}$. The conformal structure $C_{0}$ containing a complete metric with negative constant curvature on $\boldsymbol{R}^{n}$ can be considered as the conformal structure induced by an embedding of $\boldsymbol{R}^{n}$ onto an open round $n$-disk of $S^{n}$, and this embedding can be viewed as a developing map $\Phi_{0}$ of $C_{0}$. We may regard $\Phi_{0}$ as an embedding of $\boldsymbol{R}^{n}$ onto the open unit disk of $\boldsymbol{R}^{n}=S^{n} \backslash$ (a point). Composing a linear diffeomorphism of $\boldsymbol{R}^{n}$ to $\Phi_{0}$, we obtain another embedding $\Phi$ of $\boldsymbol{R}^{n}$ into $\boldsymbol{R}^{n}$. This embedding $\Phi$ can be considered as a developing map, and it defines a conformal structure $C$ on $\boldsymbol{R}^{n}$. By our construction, $d\left(\left[C_{0}\right],[C]\right)$ is finite. Now the boundary $\partial \Phi\left(\boldsymbol{R}^{n}\right)$ of $\Phi\left(\boldsymbol{R}^{n}\right)$ is a smooth regular submanifold of $\boldsymbol{R}^{n}$ with induced conformal structure, and it is diffeomorphic to $S^{n-1}$. By Liouville's theorem, each element of $\operatorname{Conf}\left(\boldsymbol{R}^{n}, C\right)$ can be extended to an element of $\operatorname{Conf}\left(\partial \Phi\left(\boldsymbol{R}^{n}\right)\right)$. It is easy to see that this extension defines a homeomorphism between these conformal transformation groups. Unless $\partial \Phi\left(\boldsymbol{R}^{n}\right)$ is a round sphere, $\operatorname{Conf}\left(\partial \Phi\left(\boldsymbol{R}^{n}\right)\right)$ is compact by a theorem of Obata ([16]) and Lelong-Ferrand ([9], [10]). Thus, if we choose the linear diffeomorphism so that it is not conformal, then $\operatorname{Conf}\left(\boldsymbol{R}^{n}, C\right)$ must be compact. On the other hand, $\operatorname{Conf}_{H}\left(\boldsymbol{R}^{n}, C_{0}\right)$ is clearly noncompact. If $[C]$ is closed in $\mathcal{T}^{0}\left(\boldsymbol{R}^{n}\right)$, this contradicts Theorem 6.3. Thus $[C]$ is not closed.

It should be noticed that, in spite of this fact, we do not know, at least from the results in this paper, whether $d$ on $\mathcal{T}^{0}\left(\boldsymbol{R}^{n}\right)$ turns out to be a distance or not.

Remark 2. As was mentioned in the remark at the end of section 2 , it should also be examined whether the Teichmüller pseudodistance on the quotient by the action of Homeo $_{C}(M)$ becomes a complete distance. It is easy to check that our proof is also valid for such spaces. Hence, our main theorem and the theorems in sections 5 and 6 remain true if we replace $\mathcal{T}^{0}(M)$ or $\mathcal{T}^{\infty}(M)$ with the corresponding quotient by Homeo $_{C}(M)$ or Diff $C(M)$. Moreover, since $\operatorname{Homeo}_{C}(M)$ is closed for any manifold $M$, we do not need the assumption corresponding to the closedness of $\operatorname{Homeo}_{H}(M)$. The same is true for the quotient by $\operatorname{Homeo}_{0}(M)$ or $\operatorname{Diff}_{0}(M)$.

\section{Some Remarks on the EXISTEnCE of eXtremal Mappings}

As we have seen, the Teichmüller pseudodistance is actually a distance in many cases. In this final section, we examine the existence of extremal mappings; the existence of $\exp d\left(\left[C_{1}\right],\left[C_{2}\right]\right)$-qc mappings between $\left(M, C_{1}\right)$ and $\left(M, C_{2}\right)$. The existence and the uniqueness of the extremal mapping between two conformal structures on a compact surface with genus greater than one plays an important role in the classical Teichmüller theory; it also seems to be an interesting problem in our situation. We will explain this briefly at the end of this section (Remark 2).

In case $C_{1}=C_{2}$, this existence problem is viewed as the question that asks whether the Teichmüller pseudodistance is actually a distance. In fact, for some cases where we have been able to prove that $d$ is a distance, we can also prove the existence of extremal mappings between two flat conformal structures. We state them below.

Proposition 7.1. Let $M$ be a compact manifold. For any pair of flat conformal structures $C_{1}$ and $C_{2}$ on $M$, there is an $\exp d\left(\left[C_{1}\right],\left[C_{2}\right]\right)$-qc mapping between $\left(M, C_{1}\right)$ and $\left(M, C_{2}\right)$. 
Proof. By our assumption, there is a sequence $\left\{\varphi_{j}\right\}_{j=1,2, \ldots}$ of qc mappings between $\left(M, C_{1}\right)$ and $\left(M, C_{2}\right)$ such that $\lim _{j \rightarrow \infty} K\left(\varphi_{j} ;\left(M, C_{1}\right),\left(M, C_{2}\right)\right)=\exp d\left(\left[C_{1}\right],\left[C_{2}\right]\right)$. By Lemma 4.1, there is a subsequence of $\left\{\varphi_{j}\right\}$, which we denote $\left\{\varphi_{j}\right\}$ again, and the limit $C_{\infty}$ of $\left\{\varphi_{j}^{*} C_{2}\right\}$ in $\mathcal{F C}^{0}(M)$ satisfying $K\left(i d_{M} ;\left(M, C_{1}\right),\left(M, C_{\infty}\right)\right)=$ $\exp d\left(\left[C_{1}\right],\left[C_{2}\right]\right)$. By Corollary 5.3, there is a $\varphi_{\infty} \in \operatorname{Homeo}_{H}(M)$ such that $\varphi_{\infty}^{*} C_{2}=$ $C_{\infty}$. Then it is clear that $\varphi_{\infty}$ is an $\exp d\left(\left[C_{1}\right],\left[C_{2}\right]\right)$-qc mapping between $\left(M, C_{1}\right)$ and $\left(M, C_{2}\right)$.

Remark. Proposition 7.1 can be viewed as a direct consequence of a result by Lelong-Ferrand ([9]).

Proposition 7.2. Suppose there is a closed point $[C]$ in $\mathcal{T}^{0}(M)$. Let $\mathcal{T}_{0}^{0}(M)$ be as in Theorem 6.3. For any pair $\left[C_{1}\right],\left[C_{2}\right] \in \mathcal{T}_{0}^{0}(M)$, there is an $\exp d\left(\left[C_{1}\right],\left[C_{2}\right]\right)$-qc mapping between $\left(M, C_{1}\right)$ and $\left(M, C_{2}\right)$.

The proof of Proposition 7.2 is the same as the first paragraph of the proof of Theorem 6.3. In particular, we have

Corollary 7.3. Let $(M, C) \cong \Omega / \Gamma$ be a Kleinian manifold. Suppose that $\Gamma$ is nonelementary and that $\operatorname{Homeo}_{H}(M)$ is closed. Then, for any pair $\left[C_{1}\right],\left[C_{2}\right] \in$ $\mathcal{T}_{0}^{0}(M)$, there is an $\exp d\left(\left[C_{1}\right],\left[C_{2}\right]\right)$-qc mapping between $\left(M, C_{1}\right)$ and $\left(M, C_{2}\right)$.

Remark 1. We can prove the existence of extremal mappings for the quotient by $\operatorname{Diff}_{C}^{r}(M)$ or $\operatorname{Diff}_{0}^{r}(M)$ in the same manner at least under the assumption corresponding to the results above.

Remark 2. Fix a flat conformal structure $C$ on $M$. Suppose that, for any flat conformal structure $C^{\prime}$ on $M$, there is an extremal mapping between $(M, C)$ and $\left(M, C^{\prime}\right)$ and that the extremal mapping is unique. Then, for any $C^{\prime}$, we can associate the unique extremal mapping. The classical theory of the Teichmüller space says that if $M$ is a compact surface with genus greater than one, in which case extremal mappings are uniquely determined, then this assignment gives rise to a homeomorphism between the Teichmüller space and the "space of extremal mappings". We explain the precise meaning of this as follows. For the extremal mapping between $C$ and a representative $C^{\prime \prime}$ of $\left[C^{\prime}\right]$, we can assign a holomorphic quadratic differential on $(M, C)$, and the differential assigned to the extremal mapping between $(M, C)$ and $\left(M, C^{\prime \prime}\right)$ coincides with that assigned to the extremal mapping between $(M, C)$ and $\left(M, C^{\prime}\right)$. From this fact, we can construct a map from $\mathcal{T}(M)$ into the space $Q(M, C)$ of the holomorphic quadratic differentials. Teichmüller proved that this map is a homeomorphism onto a unit ball of $Q(M, C)$. Through this theorem of Teichmüller, we can identify $\mathcal{T}(M)$ with the unit ball of $Q(M, C)$. Also we can regard the unit ball of $Q(M, C)$ as the "space of extremal mappings". See, for example, [4] for the details.

In general, in contrast to the case of Riemann surfaces with genus greater than one, the uniqueness of the extremal mappings does not hold because there are manifolds admitting flat conformal structures with nontrivial conformal transformation groups. In spite of this fact, the "space of extremal mappings" still seems to be interesting. For example, the existence of nontrivial conformal transformations is closely related to the appearance of singularity in $\mathcal{T}(M)$. In view of this, we might be able to expect that the "space of extremal mappings" turns out to be a kind of resolution of $\mathcal{T}(M)$. At the present stage, however, we do not even know a way to describe the "space of extremal mappings" of higher dimensional manifolds. 


\section{REFERENCES}

[1] A. Borel and Harish-Chandra, Arithmetic subgroups of algebraic groups, Ann. of Math. 75 (1962), 485-535. MR 26:5081

[2] R. D. Canary, D. B. A. Epstein and P. Green, Notes on notes of Thurston, Analytical and Geometric Aspects of Hyperbolic Spaces (D. B. A. Epstein, ed.), London Math. Soc. Lecture Notes 111, Cambridge University Press, Cambridge, 1987, pp. 3-92. MR 89e:57008

[3] R. D. Edwards and R. C. Kirby, Deformations of spaces of imbeddings, Ann. of Math. 93 (1971), 63-88. MR 44:1032

[4] F. P. Gardiner, Teichmüller theory and quadratic differentials, Wiley, New York, 1987. MR 88m:32044

[5] F. W. Gehring, Rings and quasiconformal mappings in space, Trans. Amer. Math. Soc. 103 (1962), 353-393. MR 25:3166

[6] W. M. Goldman, Geometric structures on manifolds and varieties of representations, Contemporary Math. 74 (1988), 169-198. MR 90i:57024

[7] M. Itoh, Yamabe metrics and the space of conformal structures, Internat. J. Math. 2 (1991), 659-671. MR 92j:58023

[8] D. Johnson and J. J. Millson, Deformation spaces associated to compact hyperbolic manifolds, Discrete Groups in Geometry and Analysis, Papers in honor of G. D. Mostow on his sixties birthday (R. Howe, ed.), Progress in Math. vol. 67, Birkhäuser, Boston-Basel, 1987, pp. 48106. MR 88j:22010

[9] J. Lelong-Ferrand, Transformations conformes et quasi-conformes des variété riemanniennes compactes (démonstration de la conjecture de A. Lichenerowicz), Acad. Roy. Belg. Cl. Sci. Mém. Coll. 8 (2) 39 (1971). MR 48:1100

[10] _ Geometrical interpretations of scaler curvature and regularity of conformal homeomorphisms, Differential Geometry and Relativity (M. Cahen and M. Flato, eds.), Reidel, Dordrecht-Holland, 1976, pp. 91-105. MR 56:1222

[11] O. Martio, S. Rickman and J. Väisälä, Definitions for quasiregular mappings, Ann. Acad. Sci. Fenn. Ser. A I Math. 448 (1969), 1-40. MR 41:3756

[12] O. Martio and U. Srebro, Universal radius of injectivity for locally quasiconformal mappings, Israel J. Math. 29 (1978), 17-23. MR 58:11388

[13] S. Matsumoto, Foundations of flat conformal structures, Aspects of Low Dimensional Manifolds, Advanced Studies in Pure Math. 20 (1992), 167-261. MR 93m:57014

[14] J. W. Morgan, Group actions on trees and the compactification of the space of classes of $\mathrm{SO}(n, 1)$-representations, Topology $\mathbf{2 5}$ (1986), 1-33. MR 87h:20062

[15] A. Mori, On an absolute constant in the theory of quasiconformal mappings, J. Math. Soc. Japan 8 (1956), 156-166. MR 18:27e

[16] M. Obata, The conjectures on conformal transformations of Riemannian manifolds, J. Diff. Geom. 6 (1971), 247-258. MR 46:2601

[17] Y. G. Reshetnyak, Space mappings with bounded distortion, Sibirsk. Math. Zh. 8 (1967), 629-659 (Russian). MR 90d:30067

[18] _ , The Liouville theorem with minimal regularity conditions, Sibirsk. Math. Zh. 8 (1967), 835-840 (Russian).

[19] Space mappings with bounded distortion, Translations of Math. Monographs, 73, Amer. Math. Soc., Providence, 1989. MR 90d:30067

[20] S. Rickman, Quasiregular mappings, Springer-Verlag, Berlin Heidelberg, 1993. MR 95g:30026

[21] J. Väisälä, Lectures on n-dimensional quasiconformal mappings, Lecture Notes in Math. 229, Springer-Verlag, Berlin Heidelberg New York, 1971. MR 56:12260

[22] M. Vuorinen, Conformal geometry and quasiregular mappings, Lecture Notes in Math. 1319, Springer-Verlag, Berlin Heidelberg New York, 1988. MR 89k:30021

[23] H. Whitney, Elementary structure of real algebraic varieties, Ann. of Math. 66 (1957), 545556. MR 20:2342

Mathematical Institute, Tohoku University, Aoba-ku, Sendai, 980-77, Japan

E-mail address: izeki@math.tohoku.ac.jp 